\title{
Computing Integrals and Testing Code
}

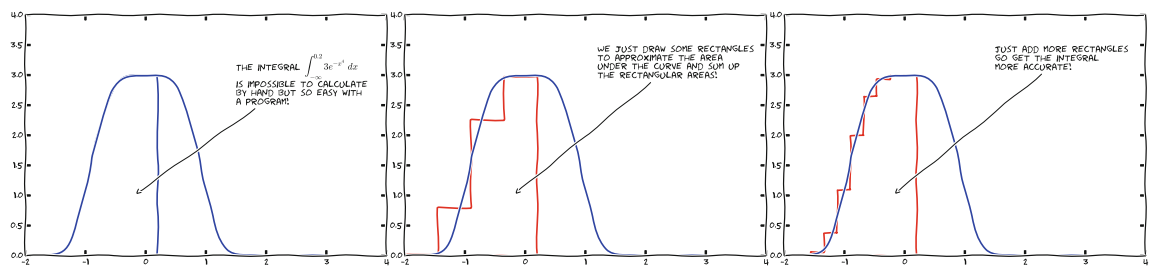

We now turn our prime attention to the solving of mathematical problems through computer programming. A fundamentally important part of programming, is to check that the written code works as intended. That is, the code must be tested. This far, we have checked our coding in rather simple ways, e.g., by comparing to hand calculations. Now, we will look at more powerful test strategies, while addressing numerical computation of integrals.

There are many reasons to choose integration as our first application. Integration is well known already from high school mathematics. Most integrals are not tractable by pen and paper, and a computerized solution approach is both very much simpler and much more powerful-you can essentially treat all integrals $\int_{a}^{b} f(x) d x$ in 10 lines of computer code!

Integration also demonstrates the difference between exact mathematics by pen and paper and numerical mathematics on a computer. The latter approaches the result of the former without any worries about rounding errors due to finite precision arithmetics in computers (in contrast to differentiation, where such errors prevent us from getting a result as accurate as we desire).

Finally, integration is thought of as a somewhat difficult mathematical concept to grasp, and programming integration should greatly improve the understanding of what integration really is and how it works.

Not only shall we understand how to use the computer to integrate, but we shall also learn a series of good habits to ensure your computer work is of the highest scientific quality. In particular, we will have a strong focus on how to write Python code that is free of programming mistakes. 
Calculating an integral is traditionally done by

$$
\int_{a}^{b} f(x) d x=F(b)-F(a)
$$

where

$$
f(x)=\frac{d F}{d x}
$$

The major problem with this procedure is that we need to find an anti-derivative $F(x)$ corresponding to a given $f(x)$. For some relatively simple integrands $f(x)$, finding $F(x)$ is a doable task. Often, however, it is really challenging, and sometimes even impossible!

The method (6.1) provides an exact or analytical value of the integral. If we relax the requirement of computing an exact value for the integral, and instead look for approximate values, produced by numerical methods, integration becomes a very straightforward task for almost any given $f(x)$ ! In particular, we do not need an anti-derivative $F(x)$ at all, since it is just the known integrand $f(x)$ that enters the calculations.

The (apparent) downside of a numerical method is that it can only find an approximate answer. Leaving the exact for the approximate is a mental barrier in the beginning, but remember that most real applications of integration will involve an $f(x)$ function that contains physical parameters, which are measured with some error. That is, $f(x)$ is very seldom exact, and it does not make sense trying to compute the integral with a smaller error than the one already present in $f(x)$.

Another advantage of numerical methods is that we can easily integrate a function $f(x)$ that is only known as samples, i.e., discrete values at some $x$ points, and not as a continuous function of $x$ expressed through a formula. This is highly relevant when $f$ is measured in a physical experiment.

\subsection{Basic Ideas of Numerical Integration}

We consider the integral

$$
\int_{a}^{b} f(x) d x
$$

Most numerical methods for computing this integral split up the original integral into a sum of several integrals, each covering a smaller part of the original integration interval $[a, b]$. This re-writing of the integral is based on a selection of integration points $x_{i}, i=0,1, \ldots, n$ that are distributed on the interval $[a, b]$. Integration points may, or may not, be evenly distributed. An even distribution simplifies expressions and is often sufficient, so we will mostly restrict ourselves to that choice. The integration points are then computed as

$$
x_{i}=a+i h, \quad i=0,1, \ldots, n,
$$


where

$$
h=\frac{b-a}{n} .
$$

That is, we get $n$ sub-intervals of the same size $h$. Given the integration points, the original integral is re-written as a sum of integrals, each integral being computed over the sub-interval between two consecutive integration points. The integral in (6.2) is thus expressed as

$$
\int_{a}^{b} f(x) d x=\int_{x_{0}}^{x_{1}} f(x) d x+\int_{x_{1}}^{x_{2}} f(x) d x+\ldots+\int_{x_{n-1}}^{x_{n}} f(x) d x .
$$

Note that $x_{0}=a$ and $x_{n}=b$.

Proceeding from (6.5), the different integration methods will differ in the way they approximate each integral on the right hand side. The fundamental idea is that each term is an integral over a small interval $\left[x_{i}, x_{i+1}\right]$, and over this small interval, it makes sense to approximate $f$ by a simple shape, say a constant, a straight line, or a parabola, that can be easily integrated. The details will become clear in the coming examples.

Computational Example To understand and compare the numerical integration methods, it is advantageous to use a specific integral for computations and graphical illustrations. In particular, we want to use an integral that we can calculate by hand such that the accuracy of the approximation methods can easily be assessed.

Our specific integral is taken from basic physics. Assume that you speed up your car from rest, on a straight road, and wonder how far you go in $T$ seconds. The displacement is given by the integral $\int_{0}^{T} v(t) d t$, where $v(t)$ is the velocity as a function of time. A rapidly increasing velocity function might be

$$
v(t)=3 t^{2} e^{t^{3}} .
$$

The distance traveled in $1 \mathrm{~s}$ is then

$$
\int_{0}^{1} v(t) d t
$$

which is the integral we aim to compute by numerical methods.

By hand, we get

$$
\int_{0}^{1} 3 t^{2} e^{t^{3}} d t=\left[e^{t^{3}}\right]_{0}^{1} \approx 1.718
$$

which is rounded to 3 decimals for convenience. 


\subsection{The Composite Trapezoidal Rule}

The integral $\int_{a}^{b} f(x) d x$ may be interpreted as the area between the $x$ axis and the graph $y=f(x)$ of the integrand. Figure 6.1 illustrates this area for the case in (6.7). Computing the integral $\int_{0}^{1} v(t) d t$ amounts to computing the area of the hatched region.

If we replace the true graph in Fig. 6.1 by a set of straight line segments, we may view the area rather as composed of trapezoids, the areas of which are easy to compute. This is illustrated in Fig. 6.2, where four straight line segments give rise to four trapezoids, covering the time intervals $[0,0.2),[0.2,0.6),[0.6,0.8)$ and $[0.8,1.0]$. Note that we have taken the opportunity here to demonstrate the computations with time intervals that differ in size.

The areas of the four trapezoids shown in Fig. 6.2 now constitute our approximation to the integral (6.7):

$$
\begin{aligned}
\int_{0}^{1} v(t) d t & \approx h_{1}\left(\frac{v(0)+v(0.2)}{2}\right)+h_{2}\left(\frac{v(0.2)+v(0.6)}{2}\right) \\
& +h_{3}\left(\frac{v(0.6)+v(0.8)}{2}\right)+h_{4}\left(\frac{v(0.8)+v(1.0)}{2}\right)
\end{aligned}
$$

where

$$
\begin{aligned}
& h_{1}=(0.2-0.0), \\
& h_{2}=(0.6-0.2)
\end{aligned}
$$

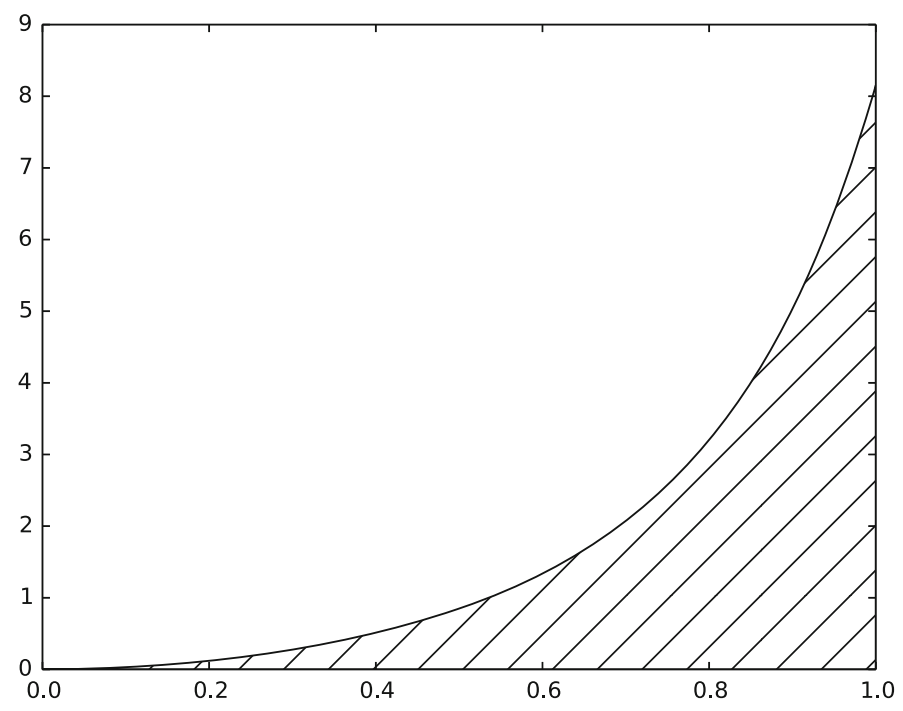

Fig. 6.1 The integral of $v(t)$ interpreted as the area under the graph of $v$ 


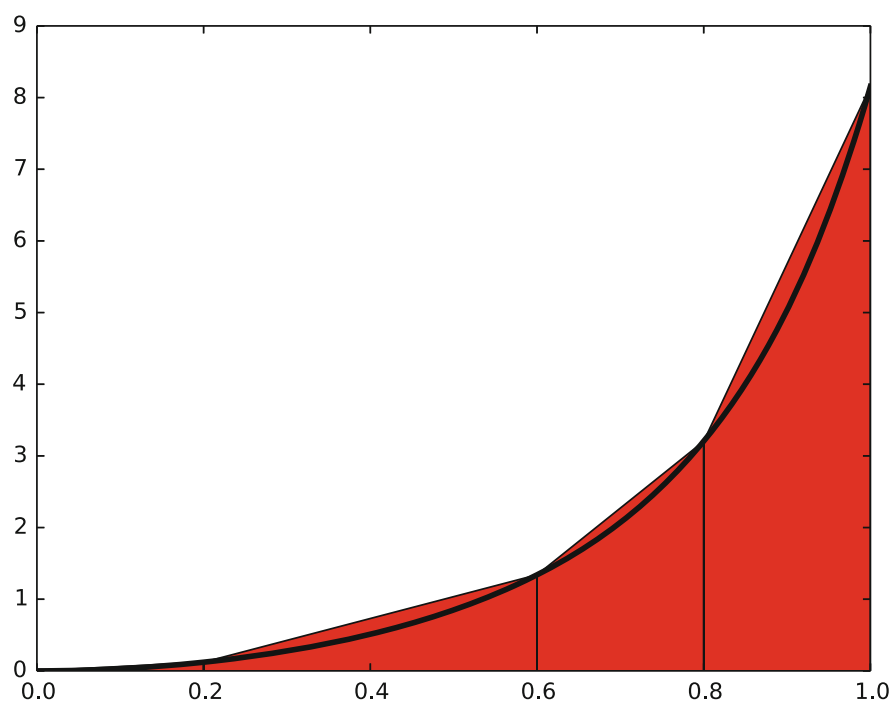

Fig. 6.2 Computing approximately the integral of a function as the sum of the areas of the trapezoids

$$
\begin{aligned}
& h_{3}=(0.8-0.6), \\
& h_{4}=(1.0-0.8)
\end{aligned}
$$

With $v(t)=3 t^{2} e^{t^{3}}$, each term in (6.9) is readily computed and our approximate computation gives

$$
\int_{0}^{1} v(t) d t \approx 1.895 .
$$

Compared to the true answer of 1.718 , this is off by about $10 \%$. However, note that we used just four trapezoids to approximate the area. With more trapezoids, the approximation would have become better, since the straight line segments at the upper trapezoid side then would follow the graph more closely. Doing another hand calculation with more trapezoids is not too tempting for a lazy human, though, but it is a perfect job for a computer! Let us therefore derive the expressions for approximating the integral by an arbitrary number of trapezoids.

\subsubsection{The General Formula}

For a given function $f(x)$, we want to approximate the integral $\int_{a}^{b} f(x) d x$ by $n$ trapezoids (of equal width). We start out with (6.5) and approximate each integral 
on the right hand side with a single trapezoid. In detail,

$$
\begin{aligned}
\int_{a}^{b} f(x) d x= & \int_{x_{0}}^{x_{1}} f(x) d x+\int_{x_{1}}^{x_{2}} f(x) d x+\ldots+\int_{x_{n-1}}^{x_{n}} f(x) d x, \\
\approx & h \frac{f\left(x_{0}\right)+f\left(x_{1}\right)}{2}+h \frac{f\left(x_{1}\right)+f\left(x_{2}\right)}{2}+\ldots+ \\
& h \frac{f\left(x_{n-1}\right)+f\left(x_{n}\right)}{2}
\end{aligned}
$$

By simplifying the right hand side of (6.15) we get

$$
\int_{a}^{b} f(x) d x \approx \frac{h}{2}\left[f\left(x_{0}\right)+2 f\left(x_{1}\right)+2 f\left(x_{2}\right)+\ldots+2 f\left(x_{n-1}\right)+f\left(x_{n}\right)\right]
$$

which is more compactly written as

$$
\int_{a}^{b} f(x) d x \approx h\left[\frac{1}{2} f\left(x_{0}\right)+\sum_{i=1}^{n-1} f\left(x_{i}\right)+\frac{1}{2} f\left(x_{n}\right)\right] .
$$

\section{Composite Integration Rules}

The word composite is often used when a numerical integration method is applied with more than one sub-interval. Strictly speaking then, writing, e.g., "the trapezoidal method", should imply the use of only a single trapezoid, while "the composite trapezoidal method" is the most correct name when several trapezoids are used. However, this naming convention is not always followed, so saying just "the trapezoidal method" may point to a single trapezoid as well as the composite rule with many trapezoids.

\subsubsection{A General Implementation}

Specific or General Implementation? Suppose we want to compute the specific integral $\int_{0}^{1} v(t) d t$, where $v(t)=3 t^{2} e^{t^{3}}$, using the (composite) trapezoidal method in (6.17). Although simple in principle, the practical steps are often confusing to many, because the notation in the abstract formulation in (6.17) differs from the notation in our special problem. Clearly, the $f, x$, and $h$ in (6.17) correspond to $v$, $t$, and perhaps $\Delta t$ for the trapezoid width in our special problem. 


\section{The Programmer's Dilemma}

1. Specific implementation: Should we write a special program for the particular integral, using the ideas from the general rule (6.17), but replacing $f$ by $v, x$ by $t$, and $h$ by $\Delta t$ ?

2. General implementation: Should we implement the general method (6.17), as written, in a general function trapezoidal ( $f$, $\mathrm{a}, \mathrm{b}, \mathrm{n}$ ) and solve the particular integral by a specialized call to this function?

A general implementation is always the best choice, not only for integrals, but when programming in general!

The first alternative in the box above sounds less abstract and therefore more attractive to many. Nevertheless, as we hope will be evident from the following, the second alternative is actually the simplest and most reliable from both a mathematical and a programming point of view.

These authors will claim that the second alternative is the essence of the power of mathematics, while the first alternative is the source of much confusion about mathematics!

General Implementation For the integral $\int_{a}^{b} f(x) d x$, computed by the formula (6.17), we want a corresponding Python function trapezoidal to take any $f, a, b$, and $n$ as input and return the approximation to the integral.

We write trapezoidal as close as possible to the formula (6.17), making sure variable names correspond to the mathematical notation:

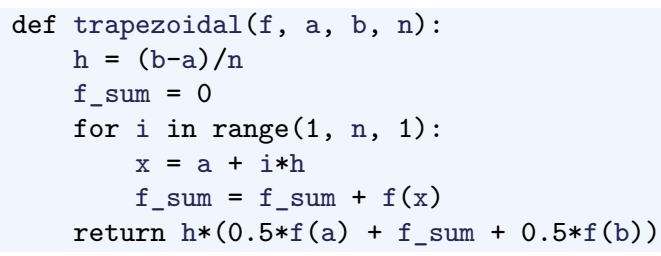

Observe how the for loop takes care of (only) the sum over $f\left(x_{i}\right)$, and that the $x$ values start with $x=a+h$ (when $i$ is 1 ), increases with $h$ for each iteration, before ending with $x=a+(n-1) h$. This is consistent with the $x$ values of the sum in (6.17). After the loop, we finalize the computation and return the result. This will be our implementation of choice for the trapezoidal function, even though, typically for programming, it could have been implemented in different ways. Which implementation to choose, is sometimes just a matter of personal taste.

One alternative version could be:

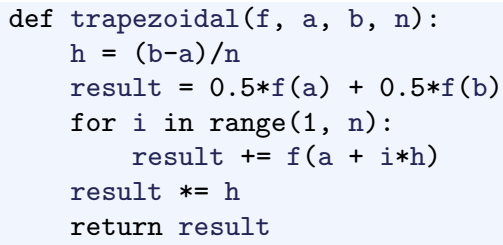


where $0.5 * f(a)+0.5 * f(b)$ is used in an initialization of result, before adding all the function evaluations $f\left(x_{i}\right)$ in the loop. After the loop, the only remaining thing, is to multiply by $\mathrm{h}$. In this second alternative, there are also a few other differences to note. The range function is called with two parameters only, giving the (default) step of 1 . In the loop, instead of computing $x=a+i * h$ prior to calling $f(x)$, we combine this into $f(a+i * h)$, which first computes $a+i * h$ and then makes the call to $f$. Also, the compact operators $+=$ and $*=$ have been used.

Using the General Implementation in a Session Having the trapezoidal function as the only content of a file trapezoidal.py automatically makes that file a module that we can import and test in an interactive session:

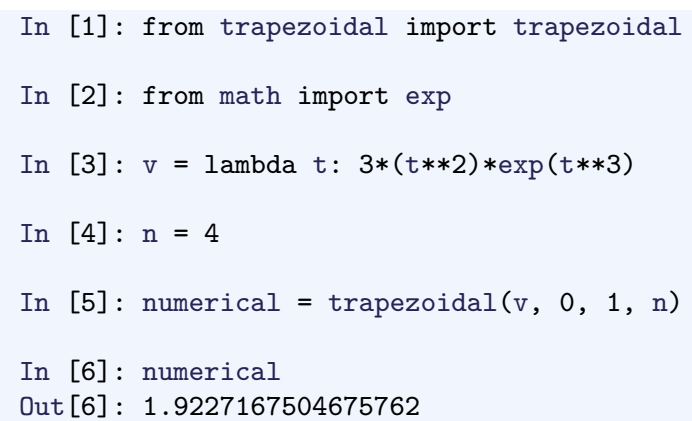

Let us compute the exact expression and the error in the approximation. Using $V$ for the anti-derivative, we get:

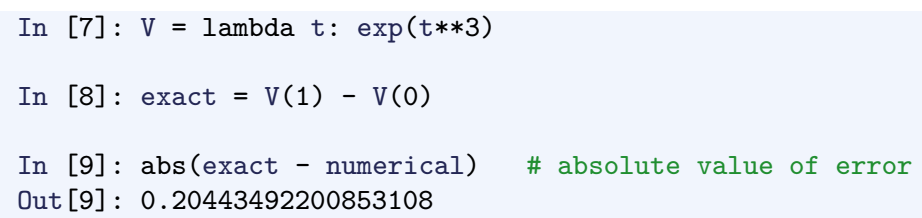

Since the sign of the error is irrelevant, we find the absolute value of the error. So, is this error convincing? We can try a larger $n$ :

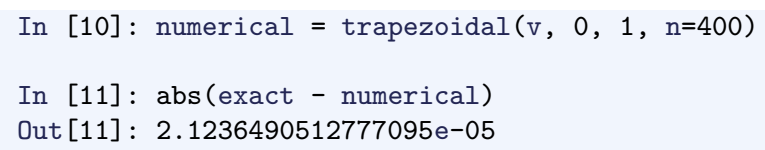

Fortunately, many more trapezoids give a much smaller error.

Using the General Implementation in a Program Instead of computing our integral in an interactive session, we can do it in a program. In that program, we need the (general) function definition of trapezoidal, and we need some code that specifies our particular integrand, as well as the other arguments required for calling trapezoidal. This code might be placed in a main program. However, it could also be placed in a function that is called from the main program. This is what we will do. A chunk of code doing a particular thing is always best isolated as a function, even if we do not see any future reason to call the function several times, and even if we have no need for arguments to parameterize what goes on inside the 
function. Thus, in the present case, we put the statements (otherwise placed a main program) inside a function named application.

To achieve flexibility, we proceed to modify trapezoidal.py, so that it has a test block and function definitions of trapezoidal and application:

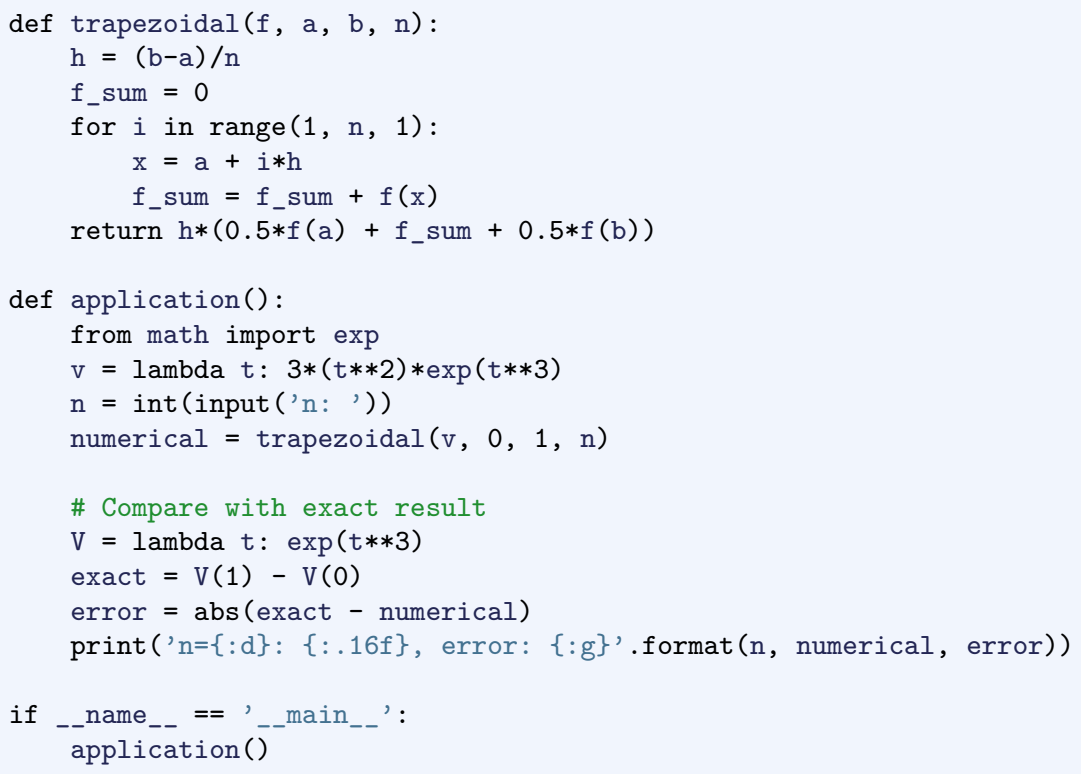

With our newly gained knowledge about the making of modules, we understand that the if test becomes true when the module file, trapezoidal.py, is run as a program, and false when the module (or part of it) is imported in another program. Consequently, with an import like from trapezoidal import trapezoidal, the test fails and application() is not called. On the other hand, if we run trapezoidal.py as a program, the test condition is positive and application() is called. A call to application implies that our special problem gets computed. The main program now gets very small, being just a single function call to application.

Running the program, e.g., with $n=4$ gives the output

$\mathrm{n}=4: 1.9227167504675762$, error: 0.204435

Clearly, with a module like the one shown here, the trapezoidal function alone (i.e., without application) can easily be imported by other programs to compute other integrals.

\subsubsection{A Specific Implementation: What's the Problem?}

Let us illustrate the implementation implied by alternative 1 in the Programmer's dilemma box in Sect.6.2.2. That is, we make a special-purpose code, where we adapt the general formula (6.17) to the specific problem $\int_{0}^{1} 3 t^{2} e^{t^{3}} d t$, in which the integrand is a velocity function $v(t)$. 
Implementation Without Function Definitions Basically, we use a for loop to compute the sum. Each term with $f(x)$ in the formula (6.17) is replaced by $3 t^{2} e^{t^{3}}$, $x$ by $t$, and $h$ by $\Delta t .^{1}$ A first version of this special implementation, without any function definition, could read

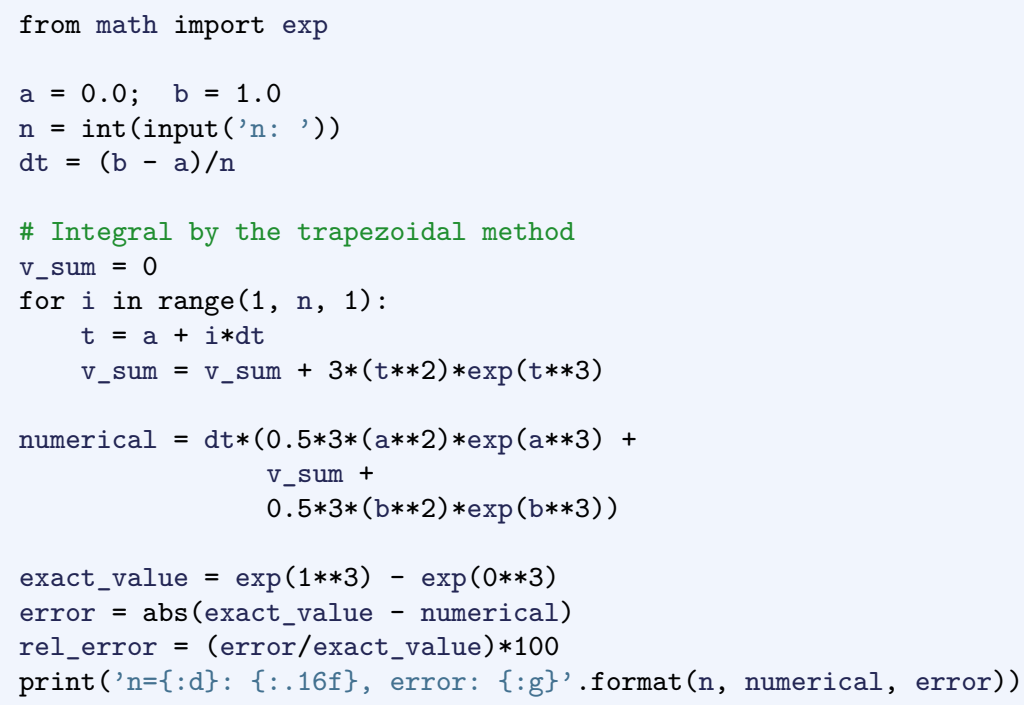

The problem with the above strategy is at least three-fold:

1. To write the code, we had to reformulate (6.17) for our special problem with a different notation. Errors come easy then.

2. To write the code, we had to insert the integrand $3 t^{2} e^{t^{3}}$ several places in the code, which quickly leads to errors.

3. If we later want to compute a different integral, the code must be edited in several places. Such edits are likely to introduce errors.

The potential errors related to point 2 serve to illustrate how important it is to define and use appropriate functions.

Implementation with Function Definitions An improved second version of the special implementation, now with functions for the integrand $\mathrm{v}$ and the antiderivative $\mathrm{V}$, might then read

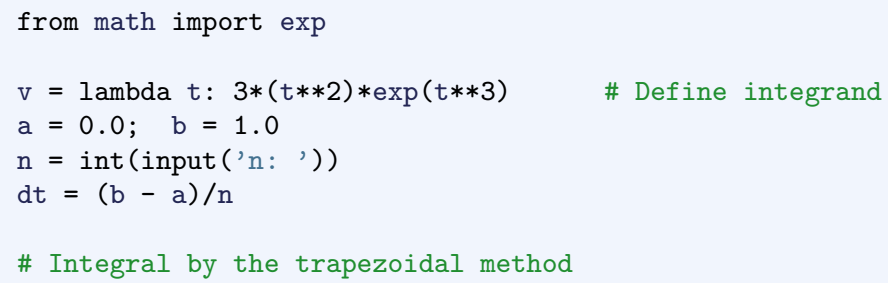

${ }^{1}$ Replacing $h$ by $\Delta t$ is not strictly required as many use $h$ as interval also along the time axis. Nevertheless, $\Delta t$ is an even more popular notation for a small time interval, so we use that here. 


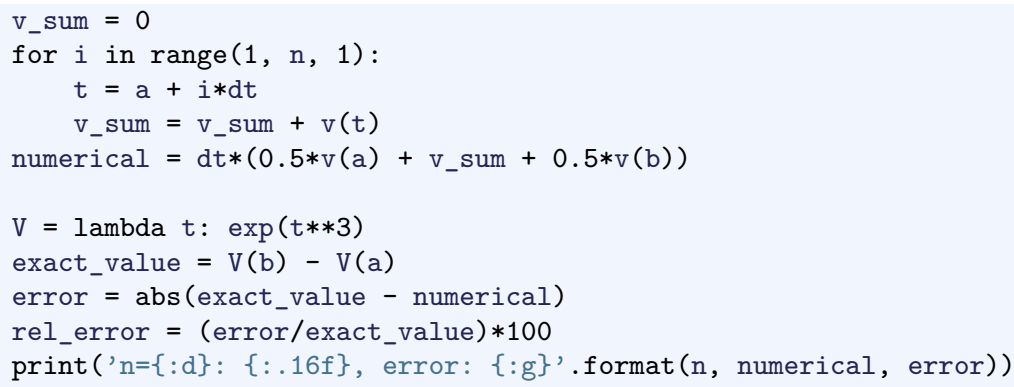

Unfortunately, the two other problems (1. and 3.) remain, and they are fundamental.

Computing Another Integral Suppose you next want to compute another integral, say $\int_{-1}^{1.1} e^{-x^{2}} d x$, using the previous specific implementation as your "starting point". What changes are required in the code then?

First of all, an anti-derivative can not (easily) be found ${ }^{2,3}$ for this new integrand, so we drop computing the integration error, and must remove the corresponding code lines. In addition,

- the notation should be changed to fit the new problem. Thus, $t$ and dt should be replaced by $\mathrm{x}$ and $\mathrm{h}$. Also, the integrand is (most likely) not a velocity any more, so the name v should be changed with, e.g., f. Similarly, v_sum should rather be f_sum then.

- the formula for $\mathrm{v}$ ( or $\mathrm{f}$ ) must be replaced by a new formula

- the limits $a$ and $b$ must be changed

These changes are straightforward to implement, but they are scattered around in the program, a fact that requires us to be very careful so we do not introduce new programming errors while we modify the code. It is also very easy to forget one or two of the required changes.

For the sake of comparison, we might see how easy it is to rather use our general implementation in trapezoidal.py for the task. With the following interactive session, it should be clear that this implementation allows us to compute the new integral $\int_{-1}^{1.1} e^{-x^{2}} d x$ without touching the implemented mathematical algorithm! We can simply do:

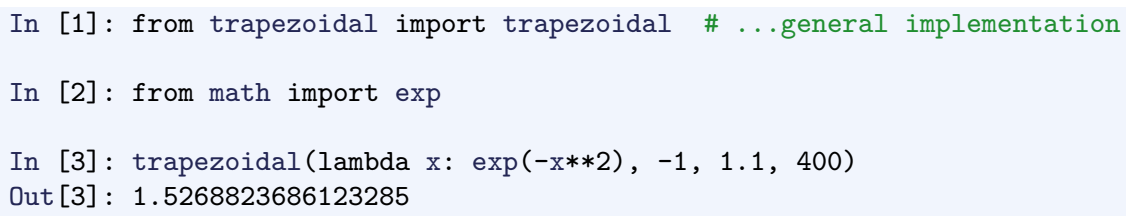

\footnotetext{
2 You cannot integrate $e^{-x^{2}}$ by hand, but this particular integral is appearing so often in so many contexts that the integral is a special function, called the Error function and written erf $(x)$. In a code, you can call erf $(x)$. The erf function is found in the math module.

${ }^{3}$ http://en.wikipedia.org/wiki/Error_function.
} 
Looking back on the two different solutions, the specific implementation and the general implementation, you should realize that implementing a general mathematical algorithm in a general function requires somewhat more abstract thinking, but the resulting code can be used over and over again! Essentially, if you apply the special-purpose style, you have to retest the implementation of the algorithm after every change of the program.

The present integral problems result in short code. In more challenging engineering problems, the code quickly grows to hundreds and thousands of lines. Without abstractions, in terms of general algorithms in general reusable functions, the complexity of the program grows so fast that it will be extremely difficult to make sure that the program works properly.

Another advantage of packaging mathematical algorithms in functions, is that a function can be reused by anyone to solve a problem by just calling the function with a proper set of arguments. Understanding the function's inner details is strictly not necessary to compute a new integral. Similarly, you can find libraries of functions on the Internet and use these functions to solve your problems without specific knowledge of every mathematical detail in the functions.

This desirable feature has its downside, of course: the user of a function may misuse it, and the function may contain programming errors and lead to wrong answers. Testing the output of downloaded functions is therefore extremely important before relying on the results.

\subsection{The Composite Midpoint Method}

The Idea Rather than approximating the area under a curve by trapezoids, we can use plain rectangles. It may sound less accurate to use horizontal lines and not skew lines following the function to be integrated, but an integration method based on rectangles (the midpoint method) is in fact slightly more accurate than the one based on trapezoids! In the midpoint method, we construct a rectangle for every sub-interval where the height equals the integrand $f$ at the midpoint of the subinterval.

For the sake of comparison, we may repeat the hand calculation of $\int_{0}^{1} v(t) d t$ in (6.7), but this time with the midpoint method. With four rectangles (Fig. 6.3) and the same sub-intervals that we used with the trapezoidal method, $[0,0.2),[0.2,0.6)$, $[0.6,0.8)$, and $[0.8,1.0]$, we get

$$
\begin{aligned}
\int_{0}^{1} v(t) d t & \approx h_{1} v\left(\frac{0+0.2}{2}\right)+h_{2} v\left(\frac{0.2+0.6}{2}\right) \\
& +h_{3} v\left(\frac{0.6+0.8}{2}\right)+h_{4} v\left(\frac{0.8+1.0}{2}\right),
\end{aligned}
$$

where $h_{1}, h_{2}, h_{3}$, and $h_{4}$ are the widths of the sub-intervals, used previously with the trapezoidal method and defined in (6.10)-(6.13).

With $v(t)=3 t^{2} e^{t^{3}}$, the approximation becomes 1.632. Compared with the true answer (1.718), this is about 5\% too small, but it is better than what we got with 


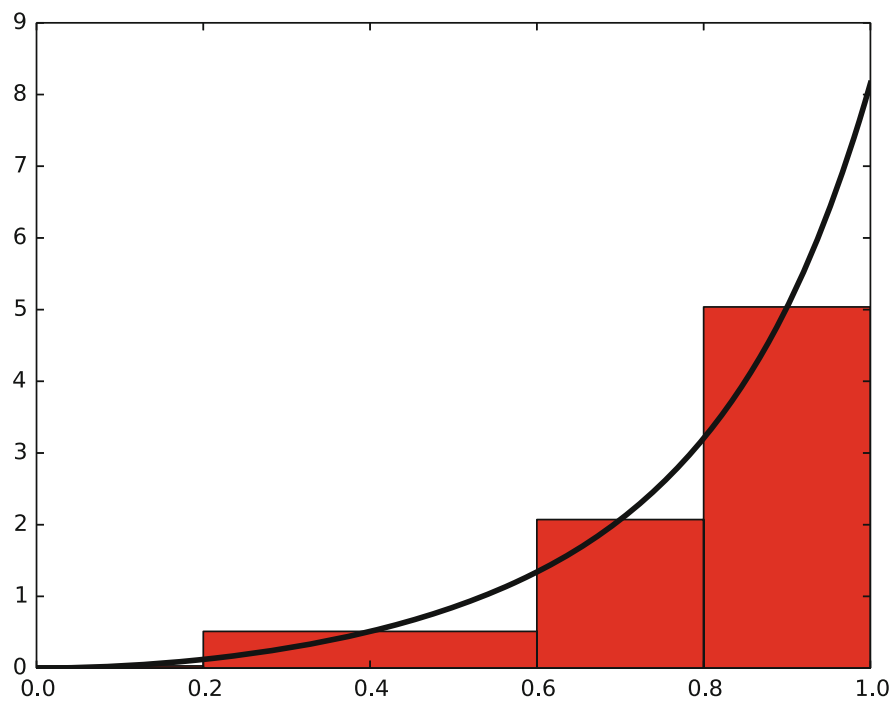

Fig. 6.3 Computing approximately the integral of a function as the sum of the areas of the rectangles

the trapezoidal method $(10 \%)$ with the same sub-intervals. More rectangles give a better approximation.

\subsubsection{The General Formula}

Let us derive a formula for the midpoint method based on $n$ rectangles of equal width:

$$
\begin{aligned}
\int_{a}^{b} f(x) d x & =\int_{x_{0}}^{x_{1}} f(x) d x+\int_{x_{1}}^{x_{2}} f(x) d x+\ldots+\int_{x_{n-1}}^{x_{n}} f(x) d x \\
& \approx h f\left(\frac{x_{0}+x_{1}}{2}\right)+h f\left(\frac{x_{1}+x_{2}}{2}\right)+\ldots+h f\left(\frac{x_{n-1}+x_{n}}{2}\right), \\
& \approx h\left(f\left(\frac{x_{0}+x_{1}}{2}\right)+f\left(\frac{x_{1}+x_{2}}{2}\right)+\ldots+f\left(\frac{x_{n-1}+x_{n}}{2}\right)\right) .
\end{aligned}
$$

This sum may be written more compactly as

$$
\int_{a}^{b} f(x) d x \approx h \sum_{i=0}^{n-1} f\left(x_{i}\right),
$$

where $x_{i}=\left(a+\frac{h}{2}\right)+i h$. 


\subsubsection{A General Implementation}

We follow the advice and lessons learned from the implementation of the trapezoidal method. Thus, we make a module midpoint.py with a general implementation of (6.20) and a function application, just like we did with the trapezoidal function:

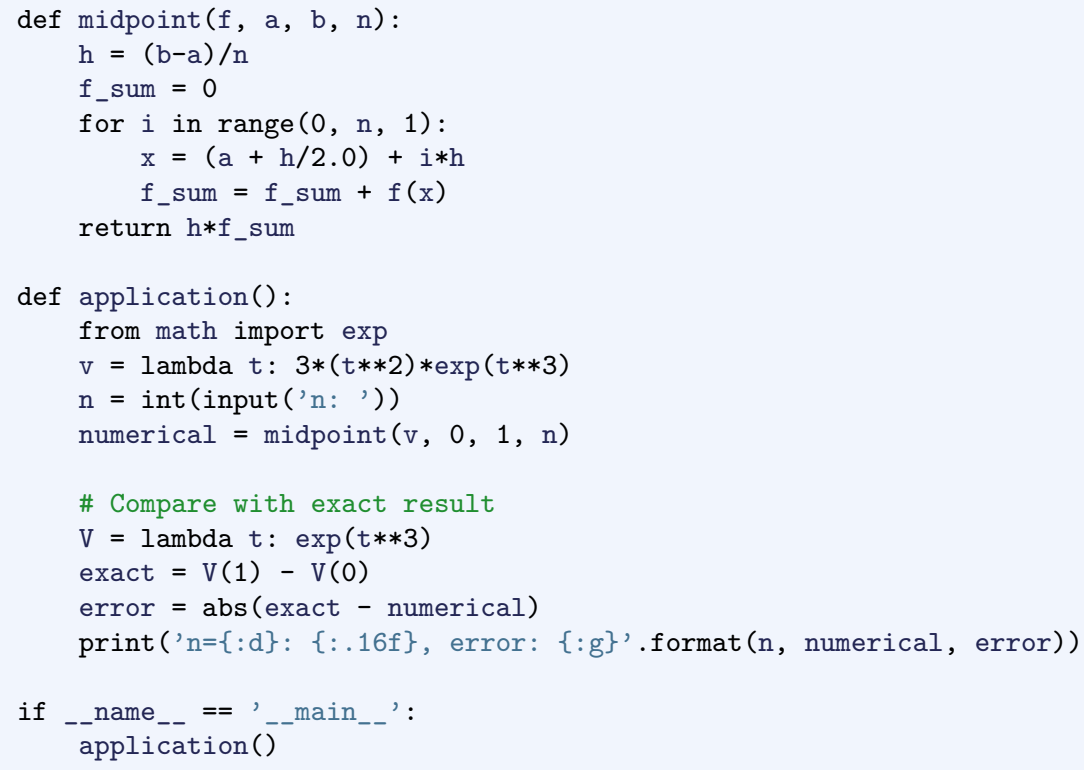

In midpoint, observe how the $x$ values in the loop start out at $x=a+\frac{h}{2}$ (when $i$ is 0 ), which is in the middle of the first rectangle. The $x$ values then increase by $h$ for each iteration, meaning that we repeatedly "jump" to the midpoint of the next rectangle as $i$ increases. This is consistent with the formula in (6.20), as is the final $x$ value of $x=a+\frac{h}{2}+(n-1) h$. To convince yourself that the first, intermediate and final $x$ values are correct, look at a case with only three rectangles, for example.

When application is called, the particular problem $\int_{0}^{1} 3 t^{2} e^{t^{3}} d t$ is computed, i.e., the same integral that we handled with the trapezoidal method. Running the program with $\mathrm{n}=4$ gives the output

$\mathrm{n}=4: 1.6189751378083810$, error: 0.0993067

The magnitude of this error is now about 0.1 in contrast to 0.2 , which we got with the trapezoidal rule. This is in fact not accidental: one can show mathematically that the error of the midpoint method is a bit smaller than for the trapezoidal method. The differences are seldom of any practical importance, and on a laptop we can easily use $n=10^{6}$ and get the answer with an error of about $10^{-12}$ in a couple of seconds. 


\subsubsection{Comparing the Trapezoidal and the Midpoint Methods}

The next example shows how easy it is to combine the trapezoidal and midpoint functions to make a comparison of the two methods. The coding is given in compare_integration_methods.py:

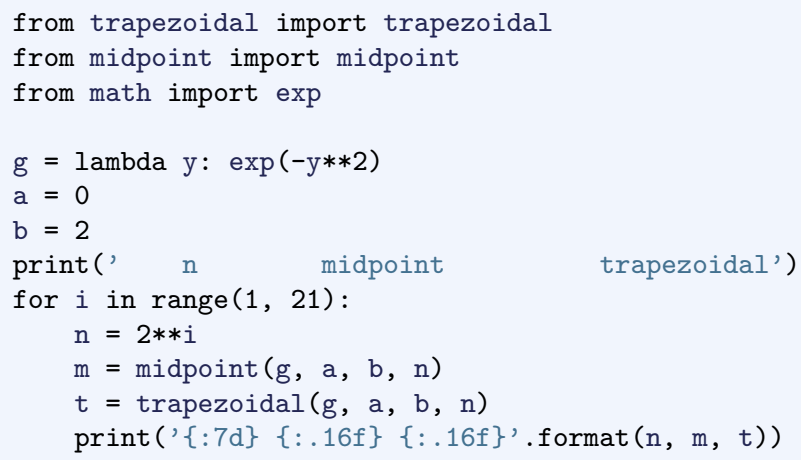

Note the efforts put into nice formatting - the output becomes

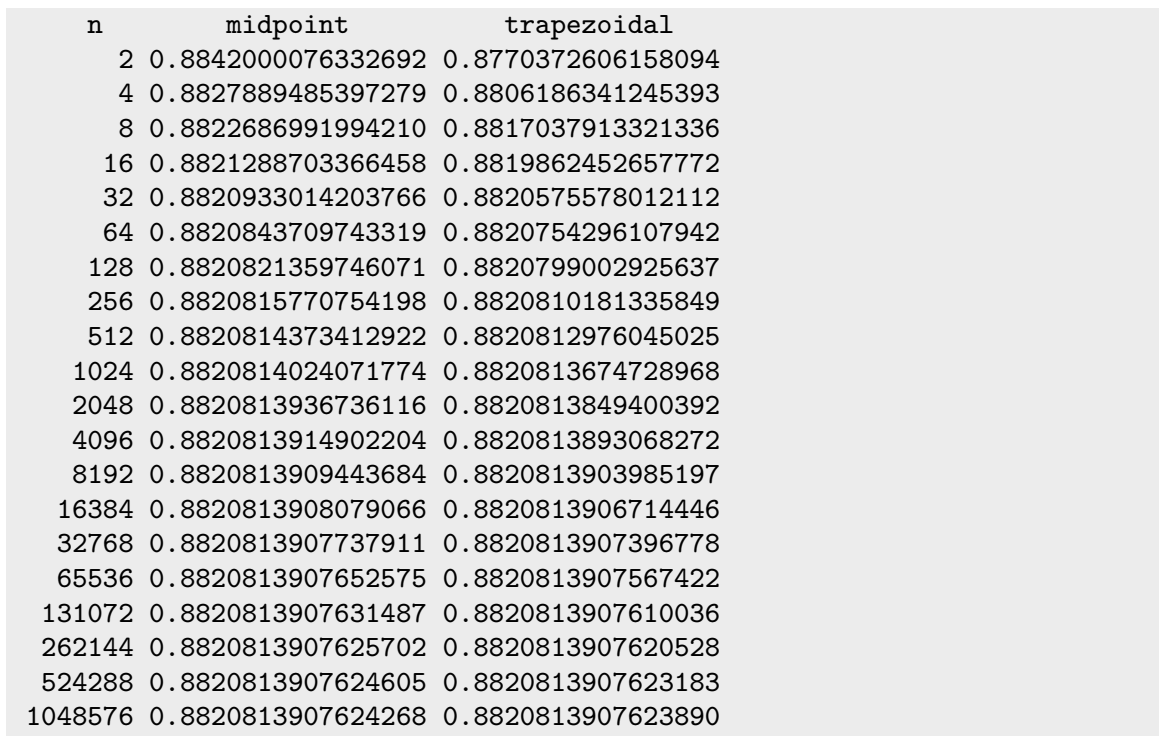

A visual inspection of the numbers shows how fast the digits stabilize in both methods. It appears that 13 digits have stabilized in the last two rows. 


\section{Remark}

The trapezoidal and midpoint methods are just two examples in a jungle of numerical integration rules. Other famous methods are Simpson's rule and Gauss quadrature. They all work in the same way:

$$
\int_{a}^{b} f(x) d x \approx \sum_{i=0}^{n-1} w_{i} f\left(x_{i}\right) .
$$

That is, the integral is approximated by a sum of function evaluations, where each evaluation $f\left(x_{i}\right)$ is given a weight $w_{i}$. The different methods differ in the way they construct the evaluation points $x_{i}$ and the weights $w_{i}$. Higher accuracy can be obtained by optimizing the location of $x_{i}$.

\subsection{Vectorizing the Functions}

The functions midpoint and trapezoidal usually run fast in Python and compute an integral to satisfactory precision within a fraction of a second. However, long loops in Python may run slowly in more complicated implementations. To increase speed, the loops can be replaced by vectorized code. The integration functions offer simple and good examples on how to vectorize loops.

We have already seen simple examples on vectorization in Sect. 1.5, when we evaluated a mathematical function $f(x)$ for a large number of $x$ values stored in an array. Basically, we can write

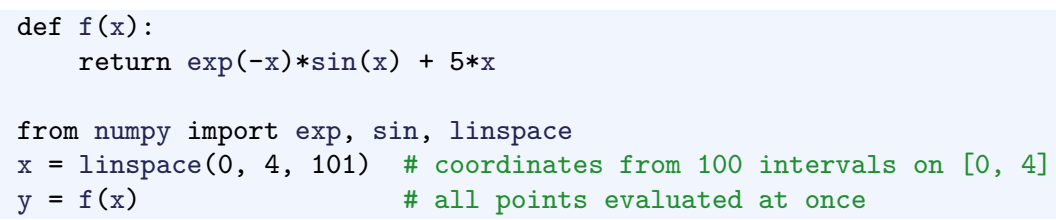

The result y is an array that, alternatively, could have been computed by running a for loop over the individual $x$ values and called $f$ for each value. Vectorization essentially eliminates this explicit loop in Python (i.e., the looping over $\mathrm{x}$ and application of $f$ to each $x$ value are instead performed in a library with fast, compiled code).

\subsubsection{Vectorizing the Midpoint Rule}

We start by vectorizing the midpoint function, since trapezoidal is not equally straightforward to vectorize. In both cases, our vectorization will remove the explicit loop. The fundamental ideas of the vectorized algorithm are to

1. compute and store all the evaluation points in one array $x$

2. call $f(x)$ to produce an array of corresponding function values

3. use the sum function to sum up the $f(x)$ values 
The evaluation points in the midpoint method are $x_{i}=a+\frac{h}{2}+i h, i=0, \ldots, n-1$. That is, $n$ uniformly distributed coordinates between $a+h / 2$ and $b-h / 2$. Such coordinates can be calculated by $\mathrm{x}=\operatorname{linspace}(\mathrm{a}+\mathrm{h} / 2, \mathrm{~b}-\mathrm{h} / 2, \mathrm{n})$. Given that the Python implementation $f$ of the mathematical function $f$ works with an array argument, which is very often the case in Python, $f(x)$ will produce all the function values in an array. The array elements are then summed up by sum, when calling $\operatorname{sum}(f(x))$. The resulting sum is to be multiplied by the rectangle width $h$ to produce the integral value. The complete function is listed below.

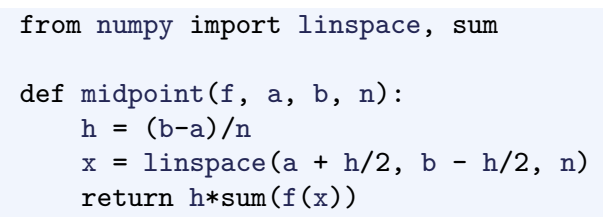

The code is found in the file integration_methods_vec.py.

Let us test the code interactively in a Python shell by computing $\int_{0}^{1} 3 t^{2} e^{t^{3}} d t$. The file with the code above has the name integration_methods_vec.py and is a valid module from which we can import the vectorized function:

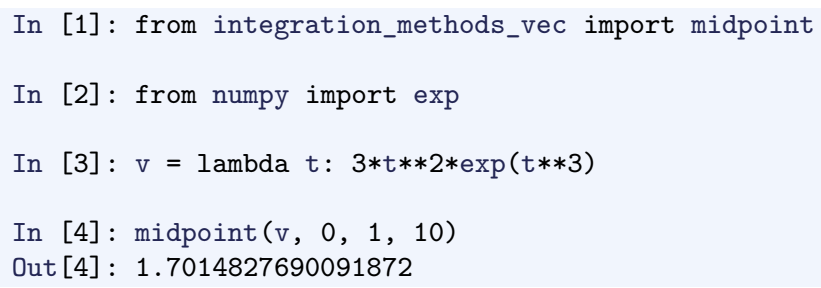

Note the necessity to use exp from numpy: our $\mathrm{v}$ function will be called with $\mathrm{x}$ as an array, and the exp function must be capable of working with an array.

The vectorized code performs all loops very efficiently in compiled code, resulting in much faster execution. Moreover, many readers of the code will also say that the algorithm looks clearer than in the loop-based implementation.

\subsubsection{Vectorizing the Trapezoidal Rule}

We can use the same approach to vectorize the trapezoidal function. However, the trapezoidal rule performs a sum where the end points have different weight. If we do $\operatorname{sum}(f(x))$, we get the end points $f(a)$ and $f(b)$ with a weight of unity instead of one half. A remedy is to subtract the error from $\operatorname{sum}(f(x))$ : $\operatorname{sum}(f(x))$ $-0.5 * f(a)-0.5 * f(b)$. The vectorized version of the trapezoidal method then becomes (the code is found in integration_methods_vec.py)

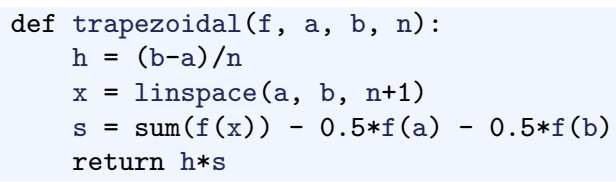




\subsubsection{Speed up Gained with Vectorization}

Now that we have created faster, vectorized versions of the functions, it is of interest to measure how much faster they are. Restricting ourselves to the midpoint method, we might proceed as:

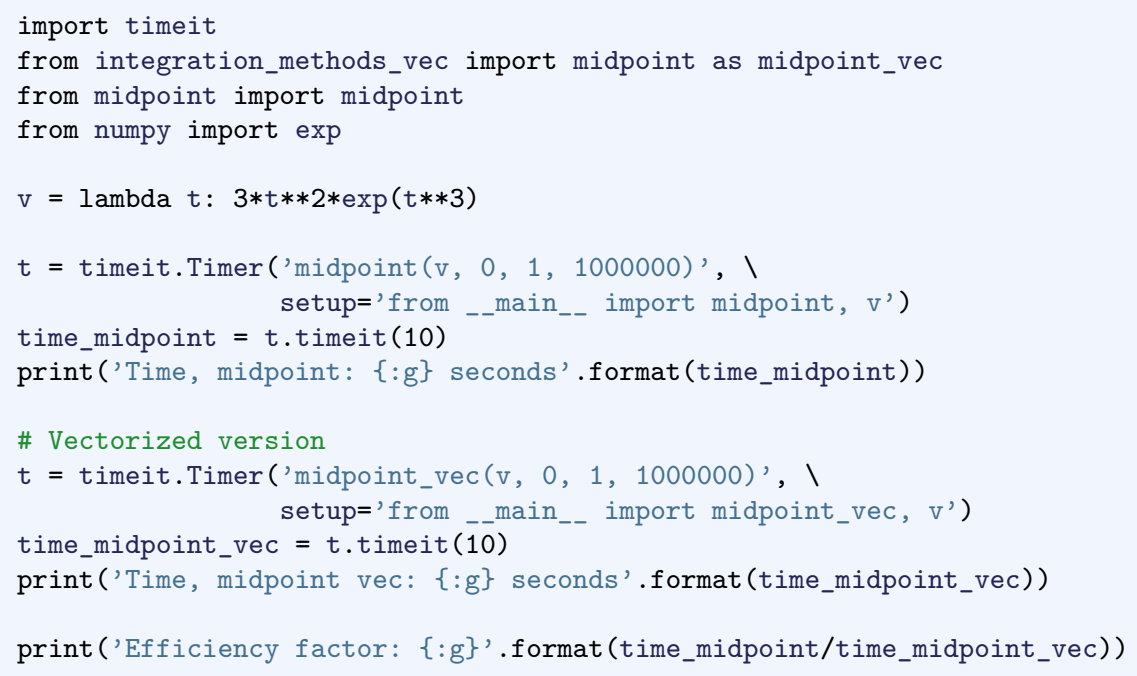

Running the program gives

Time, midpoint: 19.6083 seconds

Time, midpoint vec: 0.868379 seconds

Efficiency factor: 22.5804

We see that the vectorized version is about 20 times faster. The results for the trapezoidal method are very similar, and the factor of about 20 is independent of the number of intervals.

\subsection{Rate of Convergence}

We have seen that the numerical integration error drops when the number of subintervals $n$ is increased (causing each sub-interval to become smaller). This is fine and in line with our expectations, but some important details should be added.

Asymptotic Behavior of the Integration Error It is known that, if only the size $h$ of the sub-intervals is small enough, numerical integration methods typically give an error

$$
E=K h^{r}
$$

where $K$ is an unknown constant, while the convergence rate $r$ is a known constant that depends on the method. When a method has convergence rate $r$, it is known as an $r$-th order method. A large $r$ is beneficial, since $E$ then drops quicker when $h \rightarrow 0$. 
Clearly, when

$$
h=\frac{b-a}{n},
$$

( $n$ sub-intervals of equal size $h$ for an integration interval $[a, b]$ ), an alternative expression for $E$ follows from

$$
\begin{aligned}
E & =K h^{r}, \\
& =K\left(\frac{b-a}{n}\right)^{r}, \\
& =K(b-a)^{r}\left(\frac{1}{n}\right)^{r},
\end{aligned}
$$

which, by introducing another constant $C=K(b-a)^{r}$, gives

$$
E=C n^{-r}
$$

Convergence Rate for the Trapezoidal and Midpoint Methods Using, for example, the trapezoidal method, we may carry out some experimental runs with our test problem $\int_{0}^{1} 3 t^{2} e^{t^{3}} d t$, doubling $n$ for each run: $n=4,8,16$. The corresponding errors are then $12 \%, 3 \%$ and $0.78 \%$, respectively. These numbers indicate that the error is reduced by roughly a factor 4 when doubling $n$. Thus, it seems that the error converges to zero as $n^{-2}$, which suggests a convergence rate $r=2$. In fact, it can be shown mathematically that the trapezoidal and the midpoint method both have a convergence rate $r=2$, i.e., they are both second-order methods. Soon, we will see how this fact (and more) can be exploited in the testing of code.

\section{Remark on the Definition of Convergence Rate}

When we later address numerical solution methods for ordinary differential equations (Chap. 8), convergence rate is essentially defined like in (6.21), we just switch (not required) the symbol $h$ with $\Delta t$ (i.e., the spacing between computed solution values).

However, with iterative methods for the solving of nonlinear algebraic equations (Chap. 7), convergence rate is defined differently. In that case, one usually relates the error at an iteration to the error at the previous iteration, and the convergence rate appears as a parameter in that relation. 


\subsection{Testing Code}

\subsubsection{Problems with Brief Testing Procedures}

Previously in this book, our programs have been tested in very simple ways, usually by comparing to hand calculations. For numerical integration, in particular, testing has so far employed two strategies. When the exact solution was available, we computed the error and saw that an increase of $n$ gave a decrease in the error. When the exact solution was not available, we could (as in the comparison example of the previous section) look at the integral values and see that they stabilized as $n$ grew.

Unfortunately, these are very weak test procedures and not at all satisfactory for claiming that the software we have produced is correctly implemented.

A Deliberate Bug To see this, we can introduce a bug in the application function that calls trapezoidal: instead of integrating $3 t^{2} e^{t^{3}}$, we write "accidentally" $3 t^{3} e^{t^{3}}$, but keep the same anti-derivative $x(t)=e^{t^{3}}$ for computing the error. With the bug and $n=4$, the error is 0.1 , but without the bug the error is 0.2 ! It is of course completely impossible to tell if 0.1 is the right value of the error. Fortunately, in this case, increasing $n$ shows that the error stays about 0.3 in the program with the bug, so the test procedure with increasing $n$ (and checking that the error then decreases) points to a problem in the code.

Another Deliberate Bug Let us look at another bug, this time in the mathematical algorithm: instead of computing $\frac{1}{2}(f(a)+f(b))$ as we should, we "forget" the second $\frac{1}{2}$ and write $0.5 * f(a)+f(b)$. The error for $n=440,400$ when computing $\int_{1.1}^{1.9} 3 t^{2} e^{t^{3}} d t$ goes like $1400,107,10$, respectively, which looks promising. The problem is that the right errors should be $369,4.08$, and 0.04 . That is, the error should be reduced faster in the correct than in the buggy code. The problem, however, is that it is reduced in both codes, and we may stop further testing and believe everything is correctly implemented.

\section{Unit Testing}

A good habit is to test small pieces of a larger code individually, one at a time. This is known as unit testing: A (small) unit of the code is identified, so that a separate test for this unit can be made. The unit test should be "stand-alone" in the sense that it can be run without the outcome of other tests. Typically, one algorithm in scientific programs is considered a unit. The challenge with unit tests in numerical computing, is to deal with numerical approximation errors. A fortunate side effect of unit testing is that the programmer is forced to use functions to modularize the code into smaller, logical pieces. 


\subsubsection{Proper Test Procedures}

There are three serious ways to test the implementation of numerical methods via unit tests:

1. Comparing with hand-computed results. Relevant for problems with few arithmetic operations, i.e., small $n$.

2. Solving a problem without numerical errors. We know, for example, that the trapezoidal rule must be exact for linear integrand functions. The error produced by the program must then be zero (to machine precision).

3. Demonstrating correct convergence rates. When exact errors can be computed, a strong test is to let $n$ grow and see if the error approaches zero as fast as theory predicts. As stated previously, for the trapezoidal and midpoint rules it is known that the error depends on $n$ as $n^{-2}$ when $n \rightarrow \infty$.

\section{Remark}

When testing code, we usually choose computational problems for which the exact solution is known. This is obviously a good idea, since it allows the quality of approximate numerical answers to be judged. Do not forget, however, that the exact solution is available because we deliberately chose a problem with known exact solution. When we have finished testing (and probably fixing) the code, our belief is that the code will work also for problems with unknown exact solutions. Our strategy then, is to trust the approximate answer from our code.

Hand-Computed Results Let us use two trapezoids and compute the integral $\int_{0}^{1} v(t) d t$, where $v(t)=3 t^{2} e^{t^{3}}$ :

$$
h \frac{(v(0)+v(0.5))}{2}+h \frac{(v(0.5)+v(1))}{2}=2.463642041244344,
$$

when $h=0.5$ is the width of each trapezoid. Running the program gives exactly the same result.

Note that the exact solution is not involved here. We simply carry out the numerical algorithm by hand, "independent" from the code. We should of course get agreement between these hand calculations and program output when the same $n$ is used. However, assuming we do get agreement, that numerical answer may still differ substantially from the exact solution to the problem. That is of no concern in this test, as the aim is not to get as good an answer as possible (potentially achieved with a large $n$ ), but rather to check in a simple manner whether the algorithm "seems to be" correctly implemented.

Solving a Problem Without Numerical Errors The best unit tests for numerical algorithms involve mathematical problems where we know the numerical result beforehand. For these unit tests, we choose problems that fulfill two criteria. One 
criterion is that the exact solution is known. The other criterion is that the numerical algorithm should produce the exact solution, within machine precision, for any chosen $n$.

The second criterion may seem strange at first, but take the trapezoidal method, for example, and consider an integral like $\int_{a}^{b}(6 x-4) d x$. The integrand is here a straight line, and if you sketch it in a coordinate system along with some relevant trapezoids, you realize that the topmost side of each trapezoid comes exactly on the straight line. This is the case for whatever number $n$ of trapezoids you may choose to use. Thus, the trapezoidal method should solve that problem without any numerical error. ${ }^{4}$ We can therefore pick some linear function and construct a test function that checks for equality between the exact solution and the numerical approximation produced by our implementation of the trapezoidal method.

Note that, when testing, numbers should not just be taken out of the air. For example, a specific test case can be $\int_{1.2}^{4.4}(6 x-4) d x$. This integral involves an "arbitrary" interval [1.2, 4.4] and an "arbitrary" linear function $f(x)=6 x-4$. By "arbitrary", we mean expressions where the special numbers 0 and 1 are avoided, since these have special properties in arithmetic operations (e.g., forgetting to multiply is equivalent to multiplying by 1 , and forgetting to add is equivalent to adding 0 ).

Demonstrating Correct Convergence Rates Also for these unit tests we choose problems for which the exact solution is known. However, contrary to the previous test procedure, we now work with problems for which the numerical algorithm does not give zero approximation error. Normally, unit tests must be based on that kind of problems. Thus, the answer we get from our code will contain an approximation error, and since we know the exact solution, we may compute the size of this error. Unfortunately, this is not too helpful, since we have little chance telling if this error is what we should have got for the particular $n$ used!

Now the convergence rate comes in handy. If we know (or have reason to assume) that the numerical error has a certain asymptotic behavior when $n \rightarrow \infty$, we know at what rate the numerical error should be reduced. The idea of a corresponding unit test is then to run the algorithm for some $n$ values, compute the error (the absolute value of the difference between the exact solution and the one produced by the numerical method), and check that the error has approximately correct asymptotic behavior. For the trapezoidal and midpoint methods in particular, this means that the error should become proportional to $n^{-2}$ when $n \rightarrow \infty$.

Let us develop a more precise method for such unit tests based on convergence rates. Consider a set of $q+1$ experiments with various $n: n_{0}, n_{1}, n_{2}, \ldots, n_{q}$. We compute the corresponding errors $E_{0}, \ldots, E_{q}$. For two consecutive experiments, number $i$ and $i-1$, we have the error model

$$
\begin{aligned}
E_{i} & =C n_{i}^{-r}, \\
E_{i-1} & =C n_{i-1}^{-r} .
\end{aligned}
$$

\footnotetext{
${ }^{4}$ In fact, so would the midpoint method! This is because, for each rectangle, the error to each side of the midpoint is equally large with opposite signs, meaning that they cancel each other.
} 
These are two equations for two unknowns $C$ and $r$. We can easily eliminate $C$ by dividing, e.g., (6.26) by (6.27). Doing so, and proceeding to solve for $r$, followed by also introducing a subscript $i-1$ for $r$, gives

$$
r_{i-1}=-\frac{\ln \left(E_{i} / E_{i-1}\right)}{\ln \left(n_{i} / n_{i-1}\right)} .
$$

The subscript is introduced, since the estimated value for $r$ will vary with $i$. Hopefully, $r_{i-1}$ approaches the correct convergence rate as the number of intervals increases and $i \rightarrow q$.

\subsubsection{Finite Precision of Floating-Point Numbers}

The test procedures above lead to comparison of numbers for checking that calculations were correct. Such comparison is more complicated than what a newcomer might think. Suppose we have a calculation $a+b$ and want to check that the result is what we expect.

Adding Integers We start with $1+2$ :

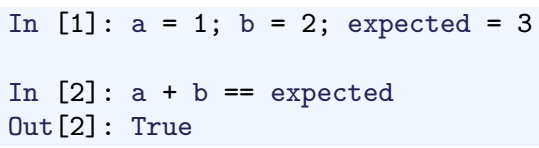

Adding Real Numbers Then we proceed with $0.1+0.2$ :

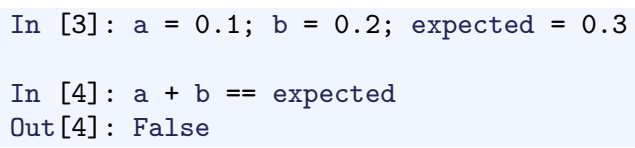

Approximate Representation of Real Numbers on a Computer So why is $0.1+$ $0.2 \neq 0.3$ ? The reason is that, generally, real numbers cannot be represented exactly on a computer. They must instead be approximated by a floating-point number 5 that can only store a finite amount of information, usually about 17 digits of a real number. Let us print $0.1,0.2,0.1+0.2$, and 0.3 with 17 decimals:

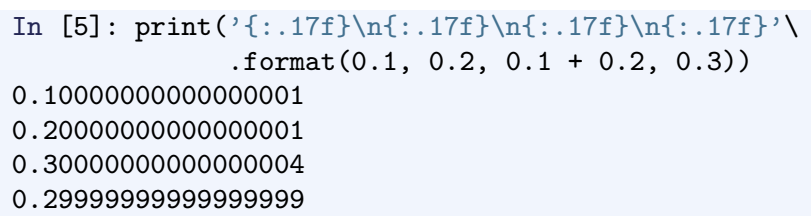

We see that all of the numbers have an inaccurate digit in the 17 th decimal place. Because $0.1+0.2$ evaluates to 0.30000000000000004 and 0.3 is represented as 0.29999999999999999 , these two numbers are not equal. In general, real numbers in Python have (at most) 16 correct decimals.

\footnotetext{
${ }^{5}$ https://en.wikipedia.org/wiki/Floating_point.
} 
Rounding Errors When we compute with real numbers, these numbers are inaccurately represented on the computer, and arithmetic operations with inaccurate numbers lead to small rounding errors in the final results. Depending on the type of numerical algorithm, the rounding errors may or may not accumulate.

Testing with a Tolerance If we cannot make tests like $0.1+0.2=0.3$, what should we then do? The answer is that we must accept some small inaccuracy and make a test with a tolerance. Here is the recipe:

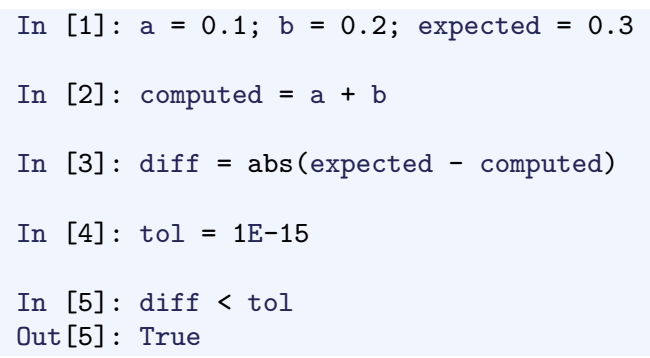

Here we have set the tolerance for comparison to $10^{-15}$, but calculating $0.3-$ $(0.1+0.2)$ shows that it equals $-5.55 e-17$, so a lower tolerance could be used in this particular example. However, in other calculations we have little idea about how accurate the answer is (there could be accumulation of rounding errors in more complicated algorithms), so $10^{-15}$ or $10^{-14}$ are robust values. As we demonstrate below, these tolerances depend on the magnitude of the numbers in the calculations.

Absolute and Relative Differences Doing an experiment with $10^{k}+0.3-\left(10^{k}+\right.$ $0.1+0.2$ ) for $k=1, \ldots, 10$ shows that the answer (which should be zero) is around $10^{16-k}$. This means that the tolerance must be larger if we compute with larger numbers. Setting a proper tolerance therefore requires some experiments to see what level of accuracy one can expect. A way out of this difficulty is to work with relative instead of absolute differences. In a relative difference we divide by one of the operands, e.g.,

$$
a=10^{k}+0.3, \quad b=\left(10^{k}+0.1+0.2\right), \quad c=\frac{a-b}{a} .
$$

Computing this $c$ for various $k$ shows a value around $10^{-16}$. A safer procedure is thus to use relative differences.

We may exemplify this in a quick session, using $k=10$,

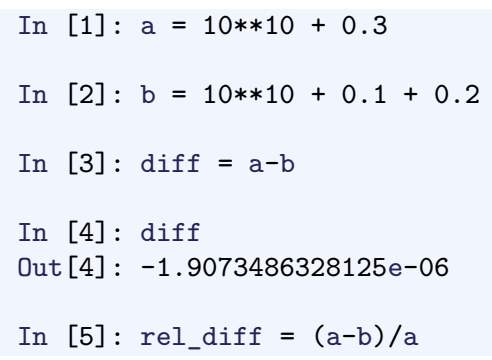


In [6]: rel_diff

Out [6] : $-1.9073486327552798 e-16$

Clearly, diff here would certainly not be smaller than a tolerance of $1 E-15$ (as we used above). So, to check whether $a$ and $b$ are equal, we should rather proceed as

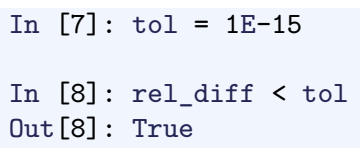

\subsubsection{Constructing Unit Tests and Writing Test Functions}

Python has several frameworks for automatic testing of software. By just one command, you can get Python to run through a (potentially) very large number of tests for various parts of your software. This is an extremely useful feature during program development: whenever you have done some changes to one or more files, launch the test command and make sure nothing is broken because of your edits.

The test frameworks nose and py . test are particularly attractive, since they are very easy to use. Tests are placed in special test functions that the frameworks can recognize and run for you. The requirements to a test function are simple:

- the name must start with test_

- the test function cannot have any arguments

- the tests inside test functions must be boolean expressions

- a boolean expression b must be tested in an assert statement of the form assert $\mathrm{b}$, msg. When $\mathrm{b}$ is true, nothing happens. However, if $\mathrm{b}$ is false, $\mathrm{msg}$ is written out, where msg is an optional object (string or number).

Suppose we have written a function

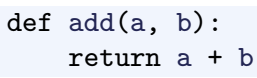

A corresponding test function can then be

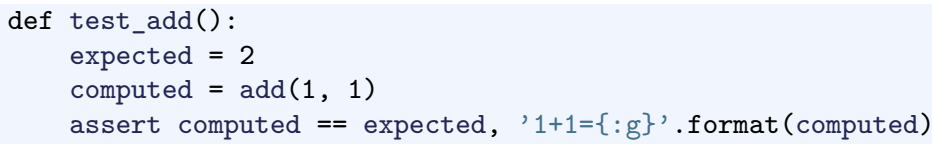

Test functions can be in any program file or in separate files, typically with names starting with test. You can also collect tests in subdirectories: running py.test $-\mathrm{s}-\mathrm{v}$ will actually run all tests in all test*.py files in all subdirectories, while nosetests $-\mathrm{s}-\mathrm{v}$ restricts the attention to subdirectories whose names start with test or end with_test or _tests.

As long as we add integers, the equality test in the test_add function is appropriate, but if we try to call add $(0.1,0.2)$ instead, we will face the rounding 
error problems explained in Sect.6.6.3, and we must use a test with tolerance instead:

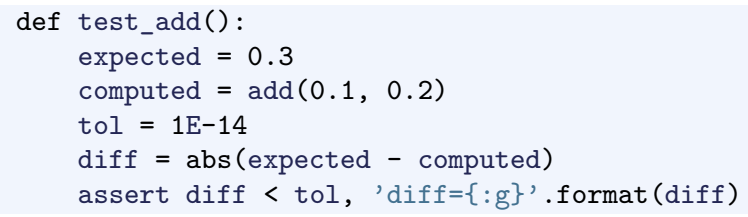

Below we shall write test functions for each of the three test procedures we suggested: comparison with hand calculations, checking problems that can be exactly solved, and checking convergence rates. We stick to testing the trapezoidal integration code and collect all test functions in one common file by the name test_trapezoidal.py.

Hand-Computed Numerical Results Our previous hand calculations for two trapezoids can be utilized in a test function like this:

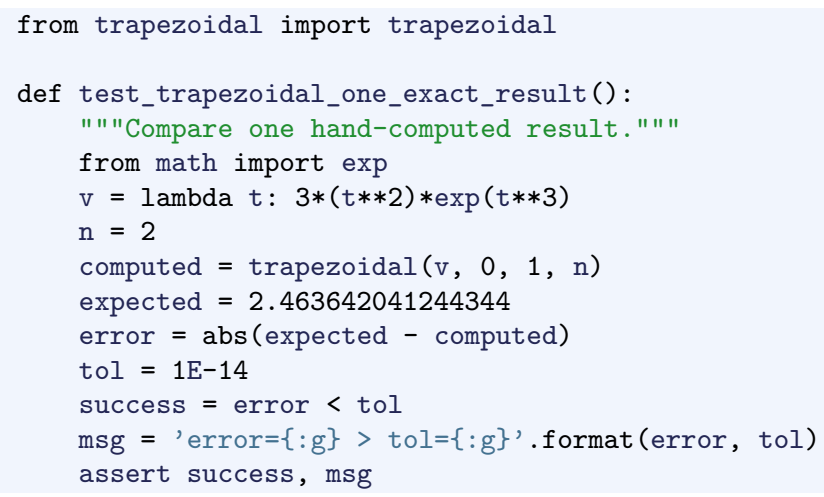

Note the importance of checking computed against expected with a tolerance: rounding errors from the arithmetics inside trapezoidal will not make the result exactly like the hand-computed one.

Solving a Problem Without Numerical Errors We know that the trapezoidal rule is exact for linear integrands. Choosing the integral $\int_{1.2}^{4.4}(6 x-4) d x$ as a test case, the corresponding test function could, for example, check with three different $n$ values, and may look like

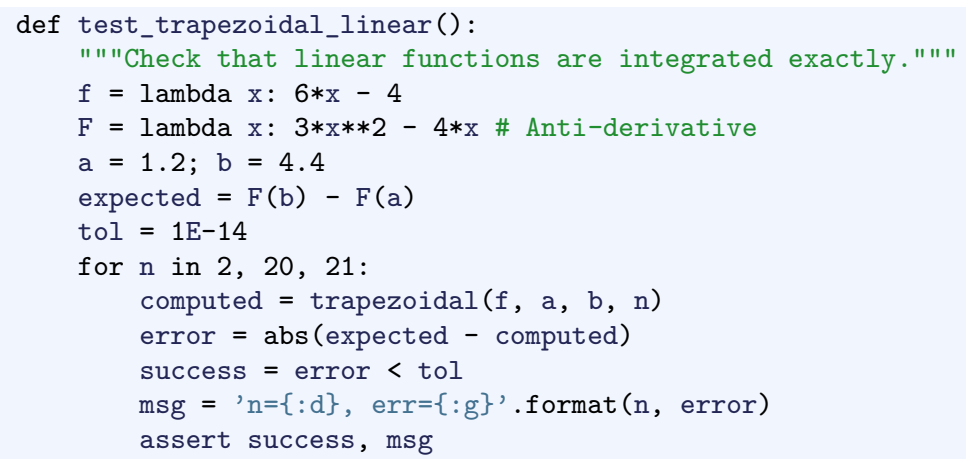


Demonstrating Correct Convergence Rates Computing convergence rates requires somewhat more tedious programming than for the previous tests, but it can be applied to more general integrands. The algorithm typically goes like

- for $i=0,1,2, \ldots, q$

$-n_{i}=2^{i+1}$

- Compute integral with $n_{i}$ intervals

- Compute the error $E_{i}$

- Estimate $r_{i}$ from (6.28) if $i>0$

The corresponding code may look like

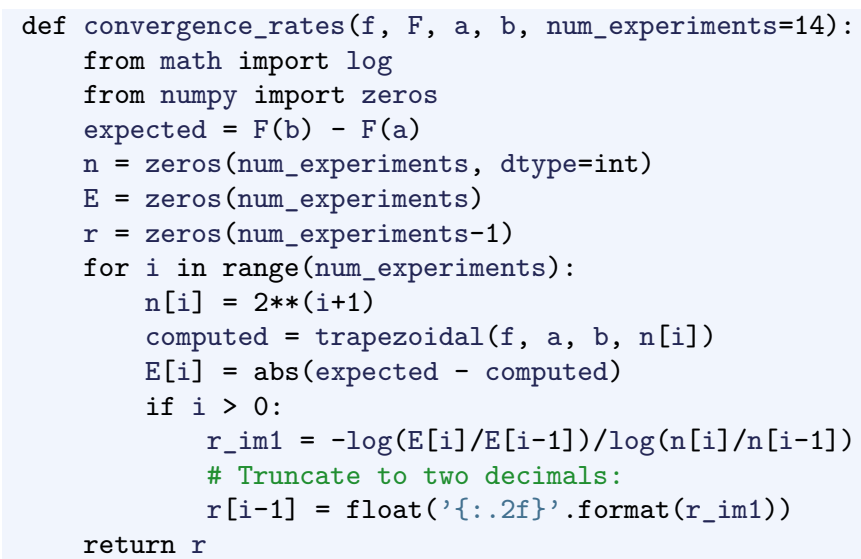

Making a test function is a matter of choosing $f, F, a$, and b, and then checking the value of $r_{i}$ for the largest $i$ :

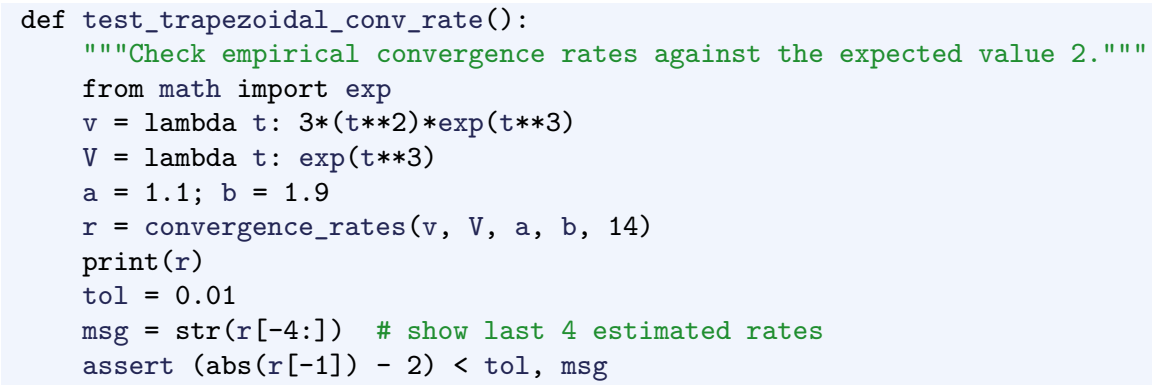

Running the test shows that all $r_{i}$, except the first one, equal the target limit 2 within two decimals. This observation suggests a tolerance of $10^{-2}$.

\subsection{Double and Triple Integrals}

\subsubsection{The Midpoint Rule for a Double Integral}

Given a double integral over a rectangular domain $[a, b] \times[c, d]$,

$$
\int_{a}^{b} \int_{c}^{d} f(x, y) d y d x
$$


how can we approximate this integral by numerical methods?

Derivation via One-Dimensional Integrals Since we know how to deal with integrals in one variable, a fruitful approach is to view the double integral as two integrals, each in one variable, which can be approximated numerically by previous one-dimensional formulas. To this end, we introduce a help function $g(x)$ and write

$$
\int_{a}^{b} \int_{c}^{d} f(x, y) d y d x=\int_{a}^{b} g(x) d x, \quad g(x)=\int_{c}^{d} f(x, y) d y .
$$

Each of the integrals

$$
\int_{a}^{b} g(x) d x, \quad g(x)=\int_{c}^{d} f(x, y) d y
$$

can be discretized by any numerical integration rule for an integral in one variable. Let us use the midpoint method (6.20) and start with $g(x)=\int_{c}^{d} f(x, y) d y$. We introduce $n_{y}$ intervals on $[c, d]$ with length $h_{y}$. The midpoint rule for this integral then becomes

$$
g(x)=\int_{c}^{d} f(x, y) d y \approx h_{y} \sum_{j=0}^{n_{y}-1} f\left(x, y_{j}\right), \quad y_{j}=c+\frac{1}{2} h_{y}+j h_{y} .
$$

The expression looks somewhat different from (6.20), but that is because of the notation: since we integrate in the $y$ direction and will have to work with both $x$ and $y$ as coordinates, we must use $n_{y}$ for $n, h_{y}$ for $h$, and the counter $i$ is more naturally called $j$ when integrating in $y$. Integrals in the $x$ direction will use $h_{x}$ and $n_{x}$ for $h$ and $n$, and $i$ as counter.

The double integral is $\int_{a}^{b} g(x) d x$, which can be approximated by the midpoint method:

$$
\int_{a}^{b} g(x) d x \approx h_{x} \sum_{i=0}^{n_{x}-1} g\left(x_{i}\right), \quad x_{i}=a+\frac{1}{2} h_{x}+i h_{x} .
$$

Putting the formulas together, we arrive at the composite midpoint method for a double integral:

$$
\begin{aligned}
\int_{a}^{b} \int_{c}^{d} f(x, y) d y d x & \approx h_{x} \sum_{i=0}^{n_{x}-1} h_{y} \sum_{j=0}^{n_{y}-1} f\left(x_{i}, y_{j}\right) \\
& =h_{x} h_{y} \sum_{i=0}^{n_{x}-1} \sum_{j=0}^{n_{y}-1} f\left(a+\frac{h_{x}}{2}+i h_{x}, c+\frac{h_{y}}{2}+j h_{y}\right) .
\end{aligned}
$$


Direct Derivation The formula (6.29) can also be derived directly in the twodimensional case by applying the idea of the midpoint method. We divide the rectangle $[a, b] \times[c, d]$ into $n_{x} \times n_{y}$ equal-sized parts called cells. The idea of the midpoint method is to approximate $f$ by a constant over each cell, and evaluate the constant at the midpoint. Cell $(i, j)$ occupies the area

$$
\left[a+i h_{x}, a+(i+1) h_{x}\right] \times\left[c+j h_{y}, c+(j+1) h_{y}\right]
$$

and the midpoint is $\left(x_{i}, y_{j}\right)$ with

$$
x_{i}=a+i h_{x}+\frac{1}{2} h_{x}, \quad y_{j}=c+j h_{y}+\frac{1}{2} h_{y} .
$$

The integral over the cell is therefore $h_{x} h_{y} f\left(x_{i}, y_{j}\right)$, and the total double integral is the sum over all cells, which is nothing but formula (6.29).

Programming a Double Sum The formula (6.29) involves a double sum, which is normally implemented as a double for loop. A Python function implementing (6.29) may look like

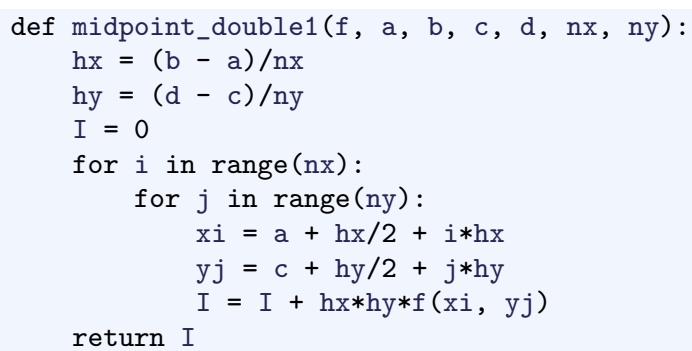

If this function is stored in a module file midpoint_double.py, we can compute some integral, e.g., $\int_{2}^{3} \int_{0}^{2}(2 x+y) d x d y=9$ in an interactive shell and demonstrate that the function computes the right number:

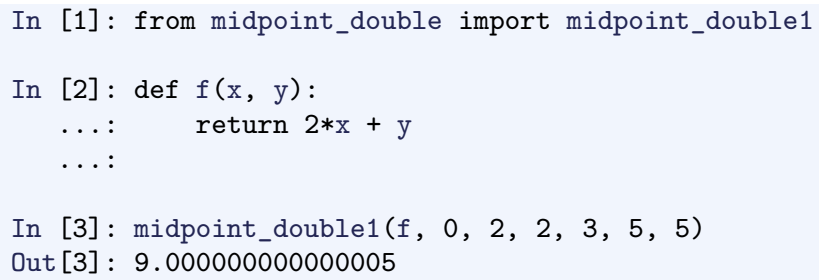

Reusing Code for One-Dimensional Integrals It is very natural to write a twodimensional midpoint method as we did in function midpoint_double1 when we have the formula (6.29). However, we could alternatively ask, much as we did in the mathematics, can we reuse a well-tested implementation for one-dimensional integrals to compute double integrals? That is, can we use function midpoint

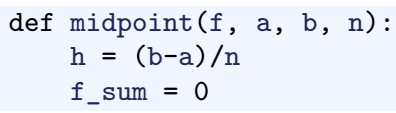




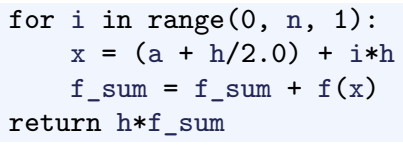

from Sect. 6.3.2 "twice"? The answer is yes, if we think as we did in the mathematics: compute the double integral as a midpoint rule for integrating $g(x)$ and define $g\left(x_{i}\right)$ in terms of a midpoint rule over $f$ in the $y$ coordinate. The corresponding function has very short code:

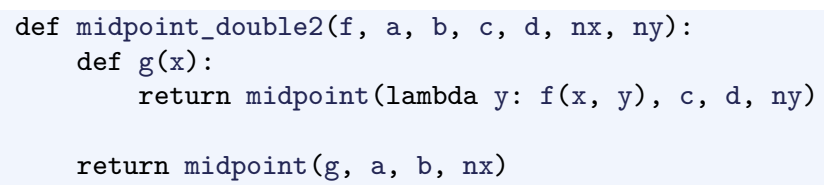

The important advantage of this implementation is that we reuse a well-tested function for the standard one-dimensional midpoint rule and that we apply the onedimensional rule exactly as in the mathematics.

Verification via Test Functions How can we test that our functions for the double integral work? The best unit test is to find a problem where the numerical approximation error vanishes because then we know exactly what the numerical answer should be. The midpoint rule is exact for linear functions, regardless of how many subinterval we use. Also, any linear two-dimensional function $f(x, y)=$ $p x+q y+r$ will be integrated exactly by the two-dimensional midpoint rule. We may pick $f(x, y)=2 x+y$ and create a proper test function that can automatically verify our two alternative implementations of the two-dimensional midpoint rule. To compute the integral of $f(x, y)$ we take advantage of SymPy to eliminate the possibility of errors in hand calculations. The test function becomes

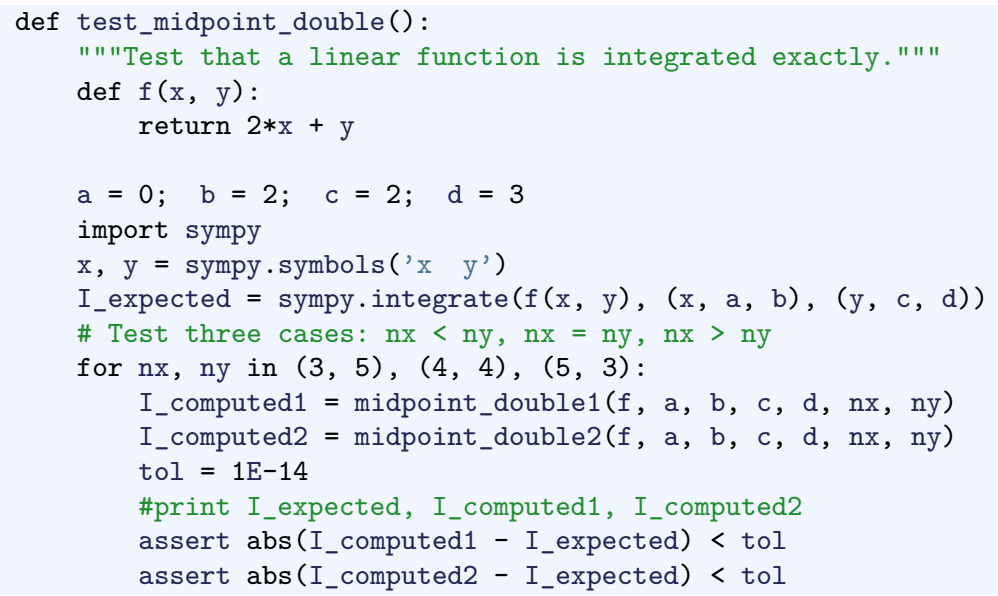




\section{Let Test Functions Speak Up?}

If we call the above test_midpoint_double function and nothing happens, our implementations are correct. However, it is somewhat annoying to have a function that is completely silent when it works-are we sure all things are properly computed? During development it is therefore highly recommended to insert a print command such that we can monitor the calculations and be convinced that the test function does what we want. Since a test function should not have any print command, we simply comment it out as we have done in the function listed above.

The trapezoidal method can be used as alternative for the midpoint method. The derivation of a formula for the double integral and the implementations follow exactly the same ideas as we explained with the midpoint method, but there are more terms to write in the formulas. Exercise 6.13 asks you to carry out the details. That exercise is a very good test on your understanding of the mathematical and programming ideas in the present section.

\subsubsection{The Midpoint Rule for a Triple Integral}

Theory Once a method that works for a one-dimensional problem is generalized to two dimensions, it is usually quite straightforward to extend the method to three dimensions. This will now be demonstrated for integrals. We have the triple integral

$$
\int_{a}^{b} \int_{c}^{d} \int_{e}^{f} g(x, y, z) d z d y d x
$$

and want to approximate the integral by a midpoint rule. Following the ideas for the double integral, we split this integral into one-dimensional integrals:

$$
\begin{aligned}
p(x, y) & =\int_{e}^{f} g(x, y, z) d z \\
q(x) & =\int_{c}^{d} p(x, y) d y \\
\int_{a}^{b} \int_{c}^{d} \int_{e}^{f} g(x, y, z) d z d y d x & =\int_{a}^{b} q(x) d x
\end{aligned}
$$


For each of these one-dimensional integrals we apply the midpoint rule:

$$
\begin{aligned}
p(x, y)=\int_{e}^{f} g(x, y, z) d z & \approx \sum_{k=0}^{n_{z}-1} g\left(x, y, z_{k}\right), \\
q(x)=\int_{c}^{d} p(x, y) d y & \approx \sum_{j=0}^{n_{y}-1} p\left(x, y_{j}\right), \\
\int_{a}^{b} \int_{c}^{d} \int_{e}^{f} g(x, y, z) d z d y d x=\int_{a}^{b} q(x) d x & \approx \sum_{i=0}^{n_{x}-1} q\left(x_{i}\right),
\end{aligned}
$$

where

$$
z_{k}=e+\frac{1}{2} h_{z}+k h_{z}, \quad y_{j}=c+\frac{1}{2} h_{y}+j h_{y} \quad x_{i}=a+\frac{1}{2} h_{x}+i h_{x} .
$$

Starting with the formula for $\int_{a}^{b} \int_{c}^{d} \int_{e}^{f} g(x, y, z) d z d y d x$ and inserting the two previous formulas gives

$$
\begin{aligned}
& \int_{a}^{b} \int_{c}^{d} \int_{e}^{f} g(x, y, z) d z d y d x \approx \\
& h_{x} h_{y} h_{z} \sum_{i=0}^{n_{x}-1} \sum_{j=0}^{n_{y}-1} \sum_{k=0}^{n_{z}-1} g\left(a+\frac{1}{2} h_{x}+i h_{x}, c+\frac{1}{2} h_{y}+j h_{y}, e+\frac{1}{2} h_{z}+k h_{z}\right) .
\end{aligned}
$$

Note that we may apply the ideas under Direct derivation at the end of Sect. 6.7.1 to arrive at (6.30) directly: divide the domain into $n_{x} \times n_{y} \times n_{z}$ cells of volumes $h_{x} h_{y} h_{z}$; approximate $g$ by a constant, evaluated at the midpoint $\left(x_{i}, y_{j}, z_{k}\right)$, in each cell; and sum the cell integrals $h_{x} h_{y} h_{z} g\left(x_{i}, y_{j}, z_{k}\right)$.

Implementation We follow the ideas for the implementations of the midpoint rule for a double integral. The corresponding functions are shown below and found in the file midpoint_triple.py.

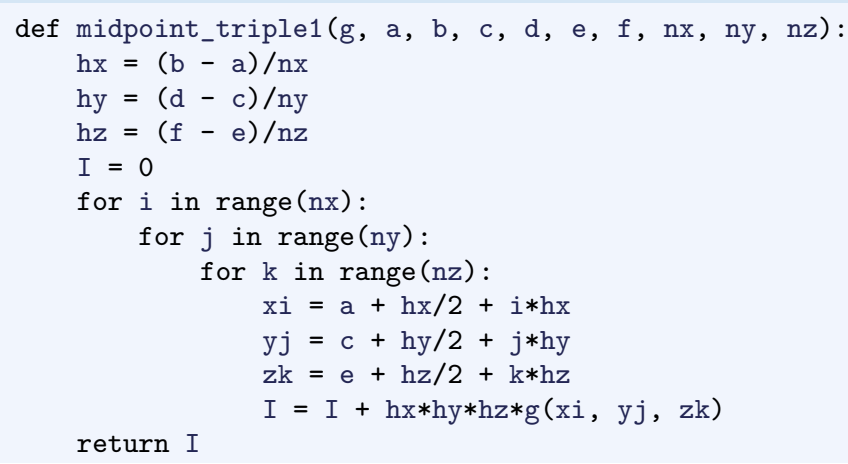




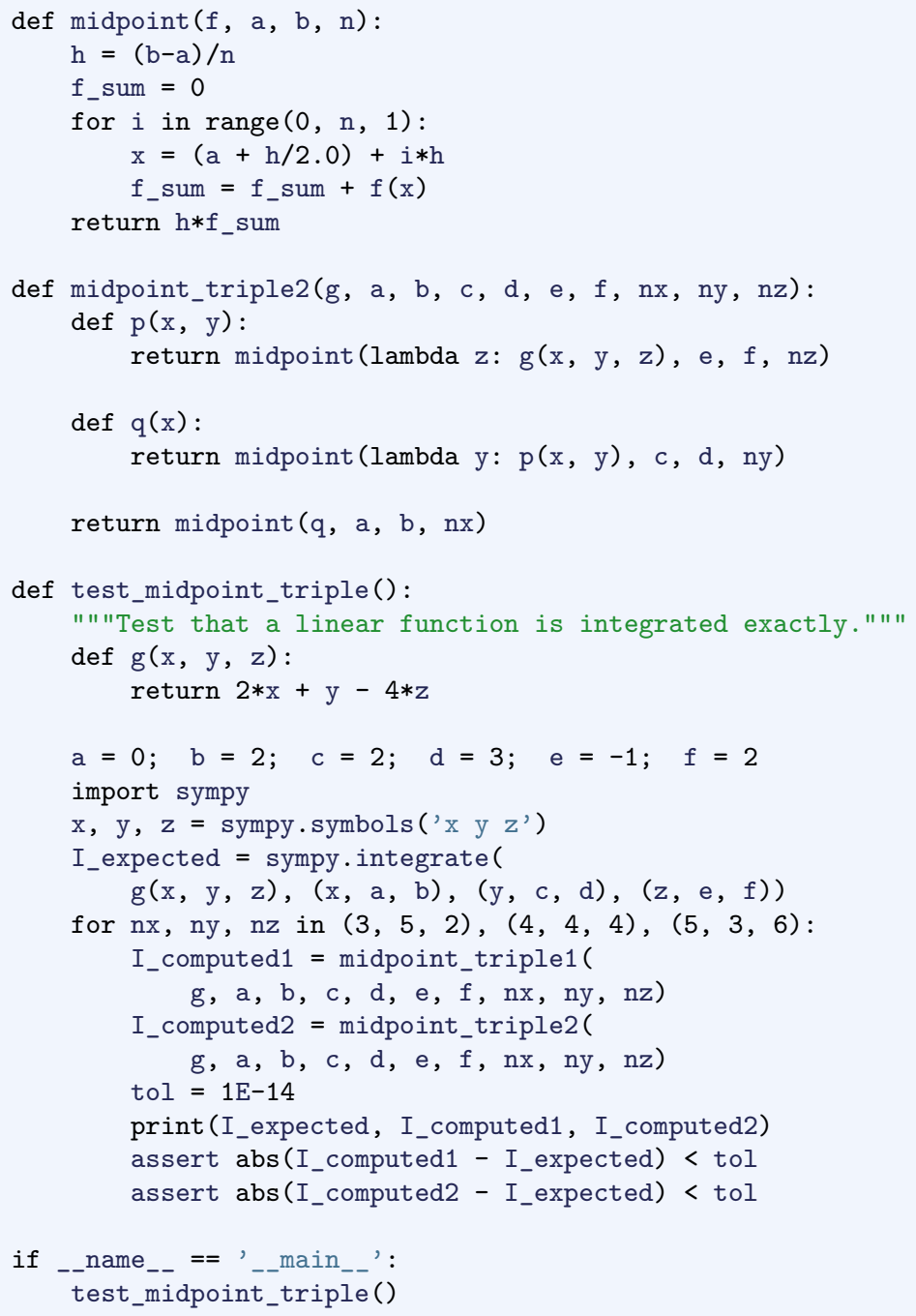

\subsubsection{Monte Carlo Integration for Complex-Shaped Domains}

Repeated use of one-dimensional integration rules to handle double and triple integrals constitute a working strategy only if the integration domain is a rectangle or box. For any other shape of domain, completely different methods must be used. A common approach for two- and three-dimensional domains is to divide the domain into many small triangles or tetrahedra and use numerical integration methods for each triangle or tetrahedron. The overall algorithm and implementation is too complicated to be addressed in this book. Instead, we shall employ an alternative, very simple and general method, called Monte Carlo integration. It can 
be implemented in half a page of code, but requires orders of magnitude more function evaluations in double integrals compared to the midpoint rule.

Monte Carlo integration, however, is much more computationally efficient than the midpoint rule when computing higher-dimensional integrals in more than three variables over hypercube domains. Our ideas for double and triple integrals can easily be generalized to handle an integral in $m$ variables. A midpoint formula then involves $m$ sums. With $n$ cells in each coordinate direction, the formula requires $n^{m}$ function evaluations. That is, the computational work explodes as an exponential function of the number of space dimensions. Monte Carlo integration, on the other hand, does not suffer from this explosion of computational work and is the preferred method for computing higher-dimensional integrals. So, it makes sense in a chapter on numerical integration to address Monte Carlo methods, both for handling complex domains and for handling integrals with many variables.

The Monte Carlo Integration Algorithm The idea of Monte Carlo integration of $\int_{a}^{b} f(x) d x$ is to use the mean-value theorem from calculus, which states that the integral $\int_{a}^{b} f(x) d x$ equals the length of the integration domain, here $b-a$, times the average value of $f, \bar{f}$, in $[a, b]$. The average value can be computed by sampling $f$ at a set of random points inside the domain and take the mean of the function values. In higher dimensions, an integral is estimated as the area/volume of the domain times the average value, and again one can evaluate the integrand at a set of random points in the domain and compute the mean value of those evaluations.

Let us introduce some quantities to help us make the specification of the integration algorithm more precise. Suppose we have some two-dimensional integral

$$
\int_{\Omega} f(x, y) d x d x
$$

where $\Omega$ is a two-dimensional domain defined via a help function $g(x, y)$ :

$$
\Omega=\{(x, y) \mid g(x, y) \geq 0\}
$$

That is, points $(x, y)$ for which $g(x, y) \geq 0$ lie inside $\Omega$, and points for which $g(x, y)<\Omega$ are outside $\Omega$. The boundary of the domain $\partial \Omega$ is given by the implicit curve $g(x, y)=0$. Such formulations of geometries have been very common during the last couple of decades, and one refers to $g$ as a levelset function and the boundary $g=0$ as the zero-level contour of the level-set function. For simple geometries one can easily construct $g$ by hand, while in more complicated industrial applications one must resort to mathematical models for constructing $g$.

Let $A(\Omega)$ be the area of a domain $\Omega$. We can estimate the integral by this Monte Carlo integration method:

1. embed the geometry $\Omega$ in a rectangular area $R$

2. draw a large number of random points $(x, y)$ in $R$ 
3. count the fraction $q$ of points that are inside $\Omega$

4. approximate $A(\Omega) / A(R)$ by $q$, i.e., set $A(\Omega)=q A(R)$

5. evaluate the mean of $f, \bar{f}$, at the points inside $\Omega$

6. estimate the integral as $A(\Omega) \bar{f}$

Note that $A(R)$ is trivial to compute since $R$ is a rectangle, while $A(\Omega)$ is unknown. However, if we assume that the fraction of $A(R)$ occupied by $A(\Omega)$ is the same as the fraction of random points inside $\Omega$, we get a simple estimate for $A(\Omega)$.

To get an idea of the method, consider a circular domain $\Omega$ embedded in a rectangle as shown below. A collection of random points is illustrated by black dots.

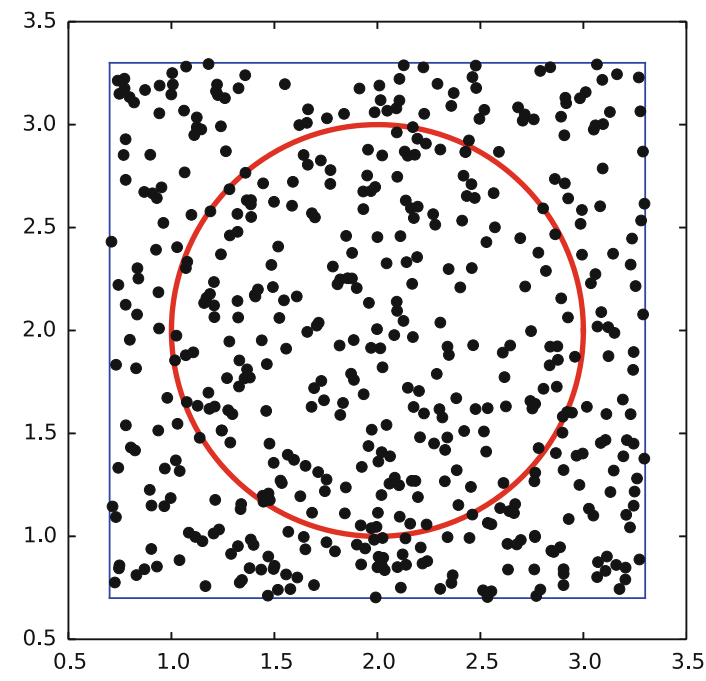

Implementation A Python function implementing $\int_{\Omega} f(x, y) d x d y$ can be written like this:

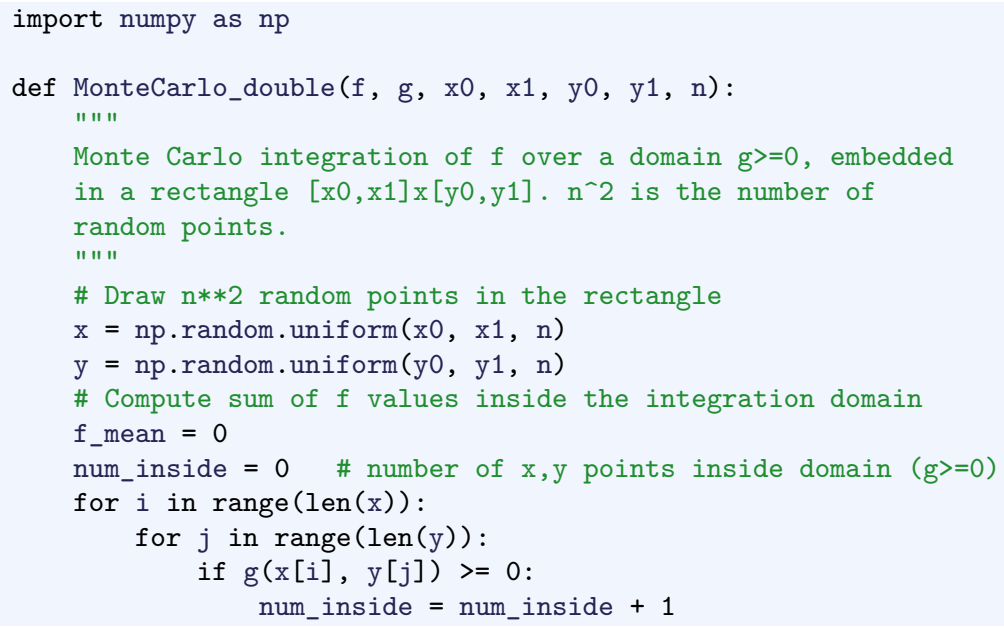




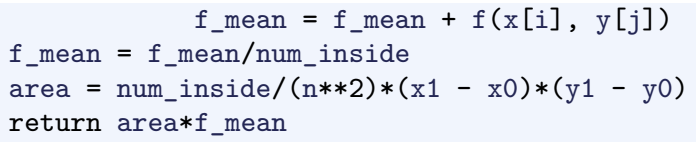

(See the file MC_double.py.)

Verification A simple test case is to check the area of a rectangle $[0,2] \times[3,4.5]$ embedded in a rectangle $[0,3] \times[2,5]$. The right answer is 3 , but Monte Carlo integration is, unfortunately, never exact so it is impossible to predict the output of the algorithm. All we know is that the estimated integral should approach 3 as the number of random points goes to infinity. Also, for a fixed number of points, we can run the algorithm several times and get different numbers that fluctuate around the exact value, since different sample points are used in different calls to the Monte Carlo integration algorithm.

The area of the rectangle can be computed by the integral $\int_{0}^{2} \int_{3}^{4.5} d y d x$, so in this case we identify $f(x, y)=1$, and the $g$ function can be specified as (e.g.) 1 if $(x, y)$ is inside $[0,2] \times[3,4.5]$ and -1 otherwise. Here is an example, using samples of different sizes, on how we can utilize the MonteCarlo_double function to compute the area:

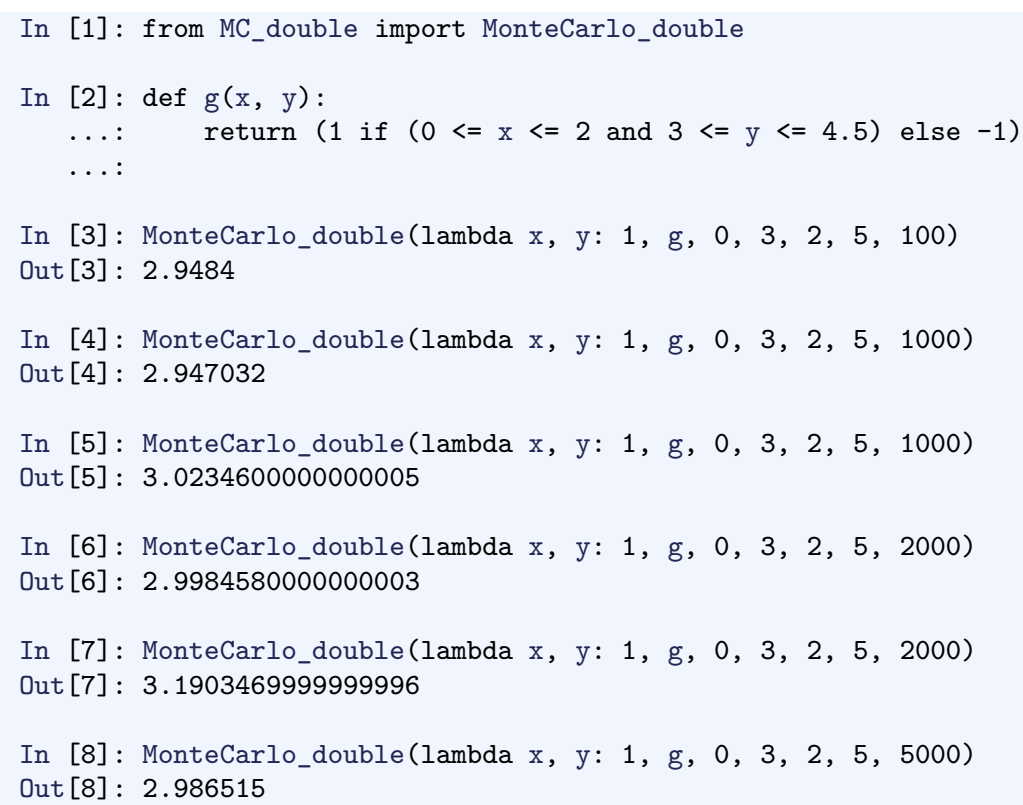

Note the compact if-else construction in the definition of $g$. It is a one-line alternative to, for example,

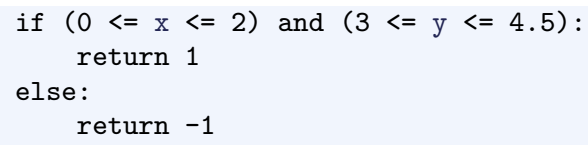

From the output, we see that the values fluctuate around 3, a fact that supports a correct implementation, but in principle, bugs could be hidden behind the inaccurate answers. 
It is mathematically known that the standard deviation of the Monte Carlo estimate of an integral converges as $n^{-1 / 2}$, where $n$ is the number of samples. This kind of convergence rate estimate could be used to verify the implementation, but the topic is beyond the scope of this book.

Test Function for Function with Random Numbers To make a test function, we need a unit test that has identical behavior each time we run the test. Thus, since the algorithm generates pseudo-random numbers, we apply the standard technique of fixing the seed (of the random number generator), so that the sequence of numbers generated is the same every time we run the algorithm. Assuming that the MonteCarlo_double function works, we fix the seed, observe a certain result, and take this result as the correct result. Provided the test function always uses this seed, we should get exactly this result every time the MonteCarlo_double function is called. Of course, this procedure does not test whether the MonteCarlo_double function works right now (but we hope our assumption of correctness is well founded!). Still, it is nevertheless useful when future changes are made, since at any time we can confirm that MonteCarlo_double gives the same answer as before. The test function can be written as shown below.

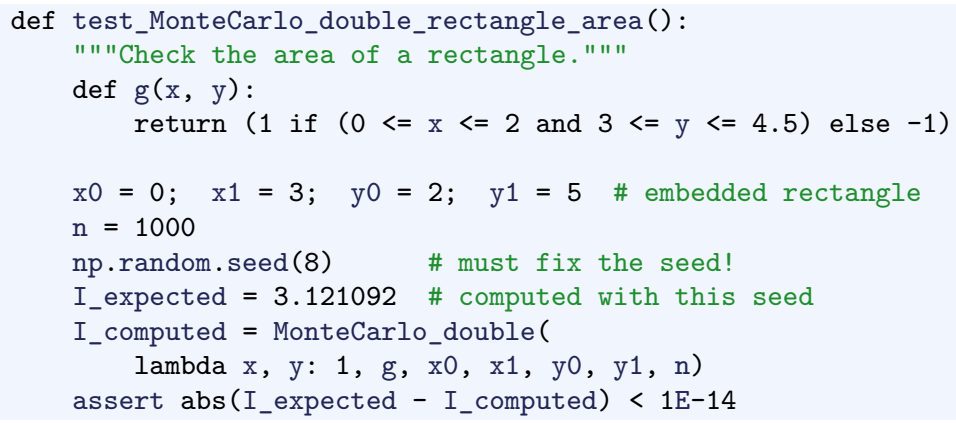

(See the file MC_double.py.)

Integral Over a Circle The test above involves a trivial function $f(x, y)=1$. We should also test a non-constant $f$ function and a more complicated domain. Let $\Omega$ be a circle at the origin with radius 2 , and let $f=\sqrt{x^{2}+y^{2}}$. This choice makes it possible to compute an exact result: in polar coordinates, $\int_{\Omega} f(x, y) d x d y$ simplifies to $2 \pi \int_{0}^{2} r^{2} d r=16 \pi / 3$. We must be prepared for quite crude approximations that fluctuate around this exact result. As in the test case above, we experience better results with larger number of points. When we have such evidence for a working implementation, we can turn the test into a proper test function. Here is an example:

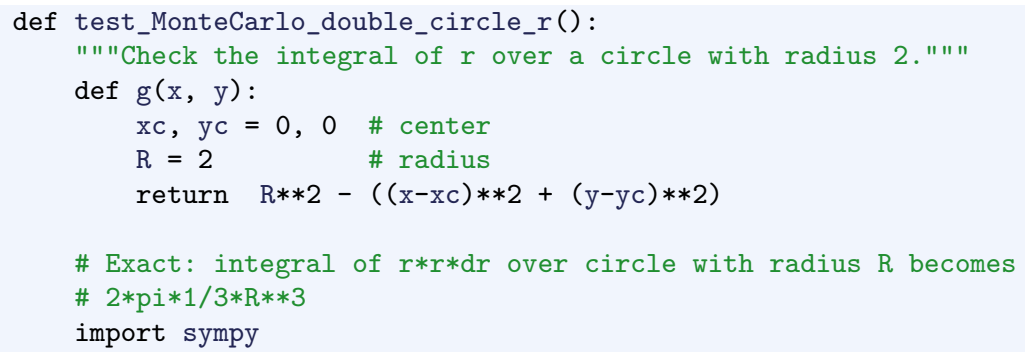




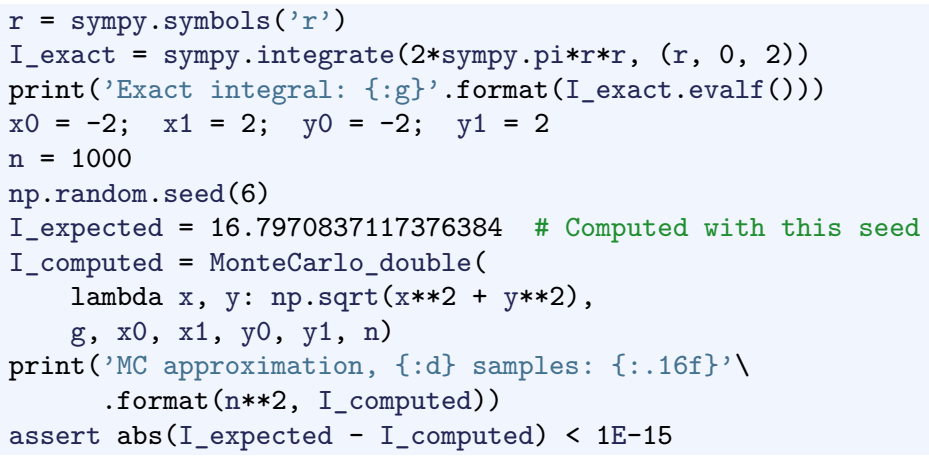

(See the file MC_double.py.)

\section{Remark About Version Control of Files}

Having a suite of test functions for automatically checking that your software works is considered as a fundamental requirement for reliable computing. Equally important is a system that can keep track of different versions of the files and the tests, known as a version control system. Today's most popular version control system is Git, ${ }^{a}$ which the authors strongly recommend the reader to use for programming and writing reports. The combination of Git and cloud storage such as GitHub is a very common way of organizing scientific or engineering work. We have a quick intro ${ }^{b}$ to Git and GitHub that gets you up and running within minutes.

The typical workflow with Git goes as follows.

1. Before you start working with files, make sure you have the latest version of them by running git pull.

2. Edit files, remove or create files (new files must be registered by git add).

3. When a natural piece of work is done, commit your changes by the git commit command.

4. Implement your changes also in the cloud by doing git push.

A nice feature of Git is that people can edit the same file at the same time and very often Git will be able to automatically merge the changes (!). Therefore, version control is crucial when you work with others or when you do your work on different types of computers. Another key feature is that anyone can at any time view the history of a file, see who did what when, and roll back the entire file collection to a previous commit. This feature is, of course, fundamental for reliable work.

\footnotetext{
${ }^{a}$ https://en.wikipedia.org/wiki/Git_(software).

${ }^{b}$ http://hplgit.github.io/teamods/bitgit/Langtangen_bitgit-bootstrap.html.
} 


\subsection{Exercises}

\section{Exercise 6.1: Hand Calculations for the Trapezoidal Method}

Compute by hand the area composed of two trapezoids (of equal width) that approximates the integral $\int_{1}^{3} 2 x^{3} d x$. Make a test function that calls the trapezoidal function in trapezoidal.py and compares the return value with the handcalculated value.

Filename: trapezoidal_test_func.py.

\section{Exercise 6.2: Hand Calculations for the Midpoint Method}

Compute by hand the area composed of two rectangles (of equal width) that approximates the integral $\int_{1}^{3} 2 x^{3} d x$. Make a test function that calls the midpoint function in midpoint. py and compares the return value with the hand-calculated value.

Filename: midpoint_test_func.py.

\section{Exercise 6.3: Compute a Simple Integral}

Apply the trapezoidal and midpoint functions to compute the integral $\int_{2}^{6} x(x-$ 1) $d x$ with 2 and 100 subintervals. Compute the error too.

Filename: integrate_parabola.py.

\section{Exercise 6.4: Hand-Calculations with Sine Integrals}

We consider integrating the sine function: $\int_{0}^{b} \sin (x) d x$.

a) Let $b=\pi$ and use two intervals in the trapezoidal and midpoint method. Compute the integral by hand and illustrate how the two numerical methods approximate the integral. Compare with the exact value.

b) Do a) when $b=2 \pi$.

Filename: integrate_sine.py.

\section{Exercise 6.5: Make Test Functions for the Midpoint Method}

Modify the file test_trapezoidal.py such that the three tests are applied to the function midpoint implementing the midpoint method for integration.

Filename: test_midpoint.py.

\section{Exercise 6.6: Explore Rounding Errors with Large Numbers}

The trapezoidal method integrates linear functions exactly, and this property was used in the test function test_trapezoidal_linear in the file test_trapezoidal.py. Change the function used in Sect.6.6.2 to $f(x)=$ $6 \cdot 10^{8} x-4 \cdot 10^{6}$ and rerun the test. What happens? How must you change the test to make it useful? How does the convergence rate test behave? Any need for adjustment?

Filename: test_trapezoidal2.py. 

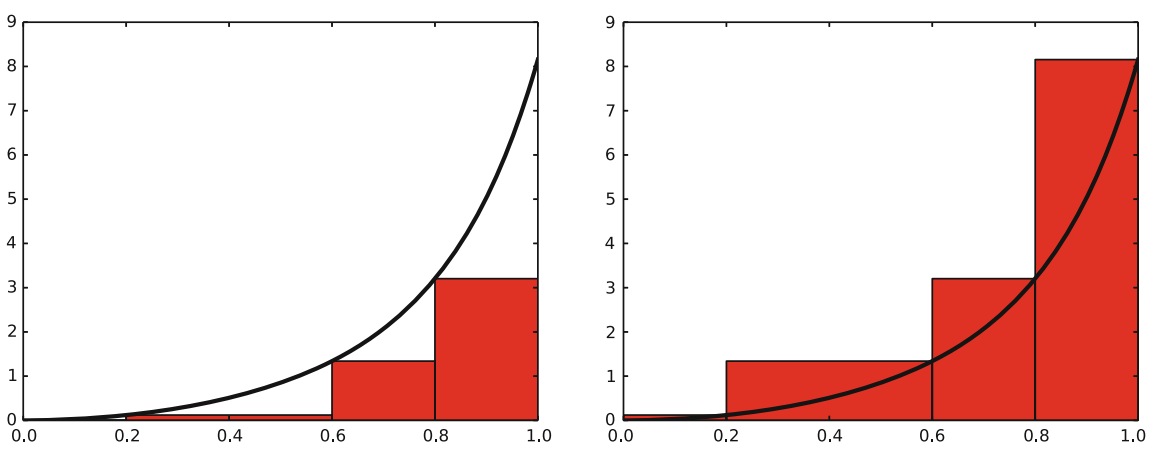

Fig. 6.4 Illustration of the rectangle method with evaluating the rectangle height by either the left or right point

Exercise 6.7: Write Test Functions for $\int_{0}^{4} \sqrt{x} d x$

We want to test how the trapezoidal function works for the integral $\int_{0}^{4} \sqrt{x} d x$. Two of the tests in test_trapezoidal.py are meaningful for this integral. Compute by hand the result of using two or three trapezoids and modify the test_trapezoidal_one_exact_result function accordingly. Then modify test_trapezoidal_conv_rate to handle the square root integral.

Filename: test_trapezoidal3.py.

Remarks The convergence rate test fails. Printing out $r$ shows that the actual convergence rate for this integral is 1.5 and not 2 . The reason is that the error in the trapezoidal method ${ }^{6}$ is $-(b-a)^{3} n^{-2} f^{\prime \prime}(\xi)$ for some (unknown) $\xi \in[a, b]$. With $f(x)=\sqrt{x}, f^{\prime \prime}(\xi) \rightarrow-\infty$ as $\xi \rightarrow 0$, pointing to a potential problem in the size of the error. Running a test with $a>0$, say $\int_{0.1}^{4} \sqrt{x} d x$ shows that the convergence rate is indeed restored to 2 .

\section{Exercise 6.8: Rectangle Methods}

The midpoint method divides the interval of integration into equal-sized subintervals and approximates the integral in each subinterval by a rectangle whose height equals the function value at the midpoint of the subinterval. Instead, one might use either the left or right end of the subinterval as illustrated in Fig. 6.4. This defines a rectangle method of integration. The height of the rectangle can be based on the left or right end or the midpoint.

a) Write a function rectangle ( $\mathrm{f}, \mathrm{a}, \mathrm{b}, \mathrm{n}$, height='left') for computing an integral $\int_{a}^{b} f(x) d x$ by the rectangle method with height computed based on the value of height, which is either left, right, or mid.

b) Write three test functions for the three unit test procedures described in Sect. 6.6.2. Make sure you test for height equal to left, right, and mid. You may call the midpoint function for checking the result when height=mid.

\footnotetext{
${ }^{6}$ http://en.wikipedia.org/wiki/Trapezoidal_rule\#Error_analysis.
} 
Hint Edit test_trapezoidal.py.

Filename: rectangle_methods.py.

\section{Exercise 6.9: Adaptive Integration}

Suppose we want to use the trapezoidal or midpoint method to compute an integral $\int_{a}^{b} f(x) d x$ with an error less than a prescribed tolerance $\epsilon$. What is the appropriate size of $n$ ?

To answer this question, we may enter an iterative procedure where we compare the results produced by $n$ and $2 n$ intervals, and if the difference is smaller than $\epsilon$, the value corresponding to $2 n$ is returned. Otherwise, we halve $n$ and repeat the procedure.

Hint It may be a good idea to organize your code so that the function adaptive_integration can be used easily in future programs you write.

a) Write a function

$$
\text { adaptive_integration( } f, a, b \text {, eps, method=midpoint) }
$$

that implements the idea above (eps corresponds to the tolerance $\epsilon$, and method can be midpoint or trapezoidal).

b) Test the method on $\int_{0}^{2} x^{2} d x$ and $\int_{0}^{2} \sqrt{x} d x$ for $\epsilon=10^{-1}, 10^{-10}$ and write out the exact error.

c) Make a plot of $n$ versus $\epsilon \in\left[10^{-1}, 10^{-10}\right]$ for $\int_{0}^{2} \sqrt{x} d x$. Use logarithmic scale for $\epsilon$.

Filename: adaptive_integration.py.

Remarks The type of method explored in this exercise is called adaptive, because it tries to adapt the value of $n$ to meet a given error criterion. The true error can very seldom be computed (since we do not know the exact answer to the computational problem), so one has to find other indicators of the error, such as the one here where the changes in the integral value, as the number of intervals is doubled, is taken to reflect the error.

\section{Exercise 6.10: Integrating $x$ Raised to $x$}

Consider the integral

$$
I=\int_{0}^{4} x^{x} d x
$$

The integrand $x^{x}$ does not have an anti-derivative that can be expressed in terms of standard functions (visit http://wolframalpha.com and type integral $(\mathrm{x} * * \mathrm{x}, \mathrm{x})$ to convince yourself that our claim is right. Note that Wolfram alpha does give you an answer, but that answer is an approximation, it is not exact. This is because Wolfram alpha too uses numerical methods to arrive at the answer, just as you will in this 
exercise). Therefore, we are forced to compute the integral by numerical methods. Compute a result that is right to four digits.

Hint Use ideas from Exercise 6.9.

Filename: integrate_x2x.py.

\section{Exercise 6.11: Integrate Products of Sine Functions}

In this exercise we shall integrate

$$
I_{j, k}=\int_{-\pi}^{\pi} \sin (j x) \sin (k x) d x
$$

where $j$ and $k$ are integers.

a) Plot $\sin (x) \sin (2 x)$ and $\sin (2 x) \sin (3 x)$ for $x \in[-\pi, \pi]$ in separate plots. Explain why you expect $\int_{-\pi}^{\pi} \sin x \sin 2 x d x=0$ and $\int_{-\pi}^{\pi} \sin 2 x \sin 3 x d x=0$.

b) Use the trapezoidal rule to compute $I_{j, k}$ for $j=1, \ldots, 10$ and $k=1, \ldots, 10$.

Filename: products_sines.py.

\section{Exercise 6.12: Revisit Fit of Sines to a Function}

This is a continuation of Exercise 4.13. The task is to approximate a given function $f(t)$ on $[-\pi, \pi]$ by a sum of sines,

$$
S_{N}(t)=\sum_{n=1}^{N} b_{n} \sin (n t) .
$$

We are now interested in computing the unknown coefficients $b_{n}$ such that $S_{N}(t)$ is in some sense the best approximation to $f(t)$. One common way of doing this is to first set up a general expression for the approximation error, measured by "summing up" the squared deviation of $S_{N}$ from $f$ :

$$
E=\int_{-\pi}^{\pi}\left(S_{N}(t)-f(t)\right)^{2} d t
$$

We may view $E$ as a function of $b_{1}, \ldots, b_{N}$. Minimizing $E$ with respect to $b_{1}, \ldots, b_{N}$ will give us a best approximation, in the sense that we adjust $b_{1}, \ldots, b_{N}$ such that $S_{N}$ deviates as little as possible from $f$. 
Minimization of a function of $N$ variables, $E\left(b_{1}, \ldots, b_{N}\right)$ is mathematically performed by requiring all the partial derivatives to be zero:

$$
\begin{gathered}
\frac{\partial E}{\partial b_{1}}=0, \\
\frac{\partial E}{\partial b_{2}}=0 \\
\vdots \\
\frac{\partial E}{\partial b_{N}}=0 .
\end{gathered}
$$

a) Compute the partial derivative $\partial E / \partial b_{1}$ and generalize to the arbitrary case $\partial E / \partial b_{n}, 1 \leq n \leq N$.

b) Show that

$$
b_{n}=\frac{1}{\pi} \int_{-\pi}^{\pi} f(t) \sin (n t) d t .
$$

c) Write a function integrate_coeffs $(f, N, M)$ that computes $b_{1}, \ldots, b_{N}$ by numerical integration, using $M$ intervals in the trapezoidal rule.

d) A remarkable property of the trapezoidal rule is that it is exact for integrals $\int_{-\pi}^{\pi} \sin n t d t$ (when subintervals are of equal size). Use this property to create a function test_integrate_coeff to verify the implementation of integrate_coeffs.

e) Implement the choice $f(t)=\frac{1}{\pi} t$ as a Python function $\mathrm{f}(\mathrm{t})$ and call integrate_coeffs $(f, 3,100)$ to see what the optimal choice of $b_{1}, b_{2}, b_{3}$ is.

f) Make a function plot_approx $(f, N, M$, filename) where you plot $f(t)$ together with the best approximation $S_{N}$ as computed above, using $M$ intervals for numerical integration. Save the plot to a file with name filename.

g) Run plot_approx (f, N, M, filename) for $f(t)=\frac{1}{\pi} t$ for $N=3,6,12,24$. Observe how the approximation improves.

h) Run plot_approx for $f(t)=e^{-(t-\pi)}$ and $N=100$. Observe a fundamental problem: regardless of $N, S_{N}(-\pi)=0$, not $e^{2 \pi} \approx 535$. (There are ways to fix this issue.)

Filename: autofit_sines.py.

\section{Exercise 6.13: Derive the Trapezoidal Rule for a Double Integral}

Use ideas in Sect.6.7.1 to derive a formula for computing a double integral $\int_{a}^{b} \int_{c}^{d} f(x, y) d y d x$ by the trapezoidal rule. Implement and test this rule.

Filename: trapezoidal_double.py. 
Exercise 6.14: Compute the Area of a Triangle by Monte Carlo Integration

Use the Monte Carlo method from Sect. 6.7.3 to compute the area of a triangle with vertices at $(-1,0),(1,0)$, and $(3,0)$.

Filename: MC_triangle.py.

Open Access This chapter is licensed under the terms of the Creative Commons Attribution 4.0 International License (http://creativecommons.org/licenses/by/4.0/), which permits use, sharing, adaptation, distribution and reproduction in any medium or format, as long as you give appropriate credit to the original author(s) and the source, provide a link to the Creative Commons licence and indicate if changes were made.

The images or other third party material in this chapter are included in the chapter's Creative Commons licence, unless indicated otherwise in a credit line to the material. If material is not included in the chapter's Creative Commons licence and your intended use is not permitted by statutory regulation or exceeds the permitted use, you will need to obtain permission directly from the copyright holder. 